

\title{
Simulating 3D periodic structures at oblique incidences with discontinuous Galerkin time-domain methods: theoretical and practical considerations
}

\author{
JONATHAN VIQUERAT ${ }^{1}$ \\ NikOlai SchmitT ${ }^{2}$ \\ Claire Scheid ${ }^{3}$
}

\author{
${ }^{1}$ Université Côte d'Azur, INRIA, CNRS, LJAD, France \\ ${ }^{2}$ Université Côte d'Azur, INRIA, CNRS, LJAD, France \\ ${ }^{3}$ Université Côte d'Azur, INRIA, CNRS, LJAD, France \\ E-mail address: claire.scheid@unice.fr.
}

\begin{abstract}
In this work, we focus on the development of the use of Periodic Boundary Conditions (PBC) with sources at oblique incidence in a nanophotonics context. In particular, we concentrate on the field transform technique used for time dependent electromagnetic wave propagation problems. We especially supplement the existing references with an analysis of the continuous model equations. Furthermore, we propose to use a Discontinuous Galerkin Time Domain (DGTD) discrete framework and study stability issues. In order to consider realistic test cases, we also provide additional details about sources, observables (reflectance, transmittance and diffraction efficiency), and the use of Complex Frequency-Shifted Perfectly-Matched Layers (CFS-PMLs). Finally, after academic numerical validations, two engineering relevant test cases are considered in the precise physical context of nanophotonics with the DioGEnES DGTD solver (http://diogenes.inria.fr).
\end{abstract}

2010 Mathematics Subject Classification. 35Q61, 65M60, 65M12.

Keywords. computational electromagnetics, time-domain Maxwell equations, discontinuous Galerkin method, periodic structures, oblique incidence sources, nanophotonics.

\section{Introduction}

A considerable amount of structures studied numerically in the nanophotonics field can be modeled using Periodic Boundary Conditions (PBC). Thus, only a unit cell of the computational domain needs to be considered, the PBC mimicking an infinite repetition of the pattern in space. Periodic structures in nano-optics cover a vast range of applications, such as color filters [13], reflectarrays for wireless communications [20], or sensing devices [9]. In many situations, a wide-band plane wave analysis at normal incidence provides enough information about the structure. However, some applications also require the knowledge of the device response for a range of incidence angles.

For a better understanding, let us suppose a $2 \mathrm{D}$ rectangular domain periodic in direction $\hat{\mathbf{x}}$ and of lateral size $\delta_{x}$. Then, suppose a plane wave propagating in the $\hat{\mathbf{z}}$ direction (i.e. at normal incidence) in the domain. In this case, the $\mathrm{PBC}$ for any field $\mathbf{U}$ can be written as:

$$
\mathbf{U}(x, z, t)=\mathbf{U}\left(x+\delta_{x}, z, t\right) .
$$

This situation is represented in figure 1.1(a), where the two matching periodic points (green dots) can update their field informations using each other's data at the same time-step. In the case of oblique incidence (figure 1.1(b)), the update of the left green dot would require information of the right green dot, which is located in an area that has not yet been hit by the incident field. In other words, this means that the field update at the left point requires the knowledge of the fields at the right point at a future time-step:

$$
\mathbf{U}(x, z, t)=\mathbf{U}\left(x+\delta_{x}, z, t+\frac{\delta_{x} \sin \theta}{c_{0}}\right)
$$




\section{J. Viquerat, N. Schmitt, et al.}

where $c_{0}$ is the speed of light in vacuum. Because of this causality issue, standard numerical methods such as Finite Difference Time Domain (FDTD) or Discontinuous Galerkin Time Domain (DGTD) applied to the governing Maxwell's equations fail to use PBC as is to simulate structures in the case of an oblique excitation. Over the years, numerous techniques have been developed in the FDTD field to deal with this modeling issue:

(1) The sine-cosine method [5], where fields are split on two separate grids corresponding to its sine- and cosine-dependent components. In this case, a generalized PBC can be used, although this restricts the computation to single-frequency sources;

(2) The sliding unit cell method [4], in which the unit cell itself is slided as the excitation progresses in the domain. This method presents several restrictions on the frequencies that can be analyzed, and several values of the field must be stored at boundaries of the domain for future updates;

(3) The multiple unit cell method [10], where several unit cells are used at the same time in order to separate the correct field propagating in the periodic structure from the error due to the causality issue. On top of requiring field storing for future updates, this method requires two unit cells in $2 \mathrm{D}$, and four in $3 \mathrm{D}$, which mitigates the interest of $\mathrm{PBC}$;

(4) The angle-updated method [16], in which the time-domain update scheme is rearranged in order to compute a part of the fields in the domain before starting the actual FDTD computation. This method suffers from a severe limitation in the incidence angles that can be used $\left(\theta<45^{\circ}\right.$ in $2 \mathrm{D}$, and $\theta<35^{\circ}$ in $\left.3 \mathrm{D}\right)$;

(5) The field-transform and the split-field techniques [14]: first, a field transformation is applied to the continuous Maxwell's equation that cancels out the causality problem. Then, for compatibility with the staggered grid inherent to Finite Difference, the fields are split before discretization. This method is well spread among the FDTD community, since it allows for any incidence angle below $90^{\circ}$ [16]. However, its counterpart is a reduction of the maximal stable time-step in the time discretization with the incidence angle (see next section).

Although a large amount of references are available for such techniques in the FDTD framework (see [17] and references therein), for the DGTD method the literature remains very shallow. To the best knowledge of the authors, the only methodological contribution on this topic is from Miller et al. [11], although the method has been exploited afterwards [19]. In [11], the authors revisit the field transform method, and then present its discretization in the DGTD framework. No analysis is provided, neither for the continuous nor for the discrete equations. Regarding domain truncation, only first-order Absorbing Boundary Conditions (ABC) are covered, and very little is said about the incident fields or the observables.

In this paper, we focus on developing the field transform technique in the context of nanophotonics periodic structures. We use a DGTD discretization framework for the discretization of the governing Maxwell's equation and provide a stability analysis. We extend the technique to incorporate the possibility of using PML's and finally, we give several numerical results from academical to more applied test cases.

In the following section, we shortly sum up the field transform technique, and the continuous system is analyzed with the method of characteristics and an energy estimate is provided. Then, its discretization with the DGTD method is covered, and a stability proof is given in this context. Along the way, insights about the transformation of sources, observables (reflectance, transmittance and diffraction efficiency), and the use of CFS-PMLs for the transformed system are given and included in 


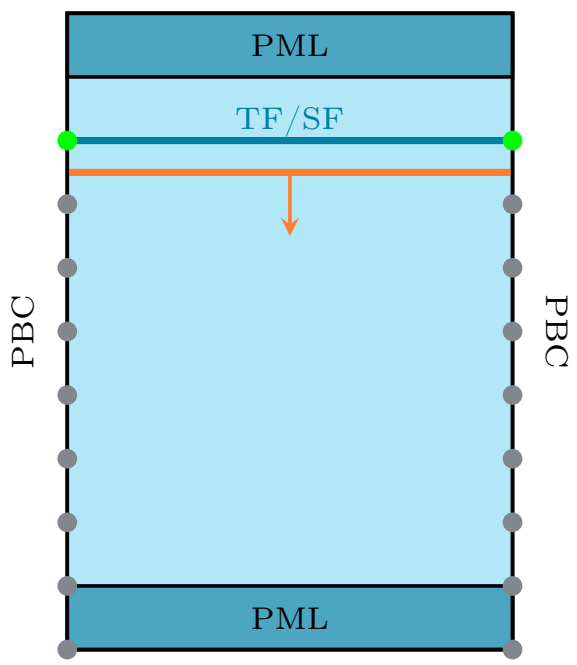

(a) Normal incidence

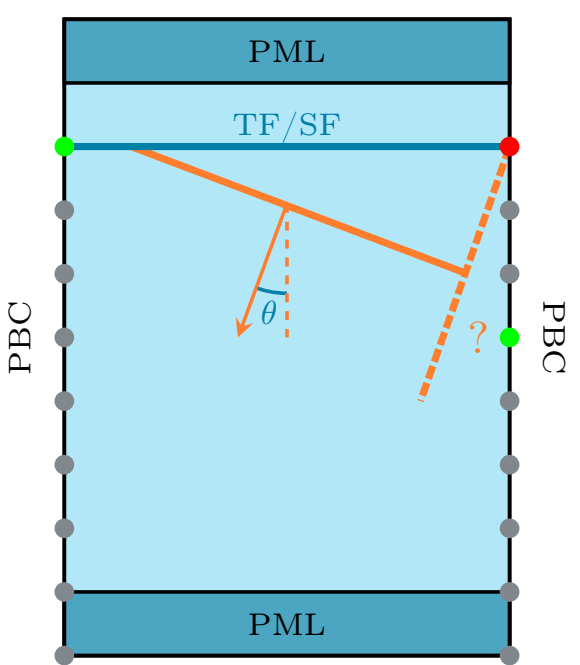

(b) Oblique incidence

FiguRE 1.1. 2D configurations of normal and oblique incidence with PBC. The domain is periodic in the lateral directions and is infinite in the vertical direction. Here the notation PML at top and bottom already refers to the artificial numerical truncation of the domain that will be used in the sequel. The incident plane wave is oriented toward the bottom of the domain. Numerically, this will be imposed at an artificial interface called the Total-Field/Scattered-Field interface (see later for details). In normal incidence, the PBC applies naturally, and at each discretization point matching information is available from the periodic neighbor point (green dots). In oblique incidence, a causality issue appears, since the matching information for the left green dot is located in a zone that has not been illuminated by the incident field yet.

the analysis. We then consider the numerical implementation and validate the latter on a textbook case for which an analytical solution is known. Finally, we consider two realistic cases: (i) a silicon-based color filter, which response is analyzed under various incidence angles, and (ii) a diffraction grating, for which we compare the diffraction efficiencies at normal and oblique incidences.

\section{The field transform technique}

\subsection{Transformed equations}

We denote by $\varepsilon_{0}, \mu_{0}, c_{0}$ respectively the permittivity, permeability and wave speed in vacuum and follow [11] for the setting of the equations. We now consider a $3 D$ setting and a plane wave of angular frequency $\omega$ defined by a wave vector $\mathbf{k}={ }^{t}\left(k_{x}, k_{y}, k_{z}\right)$ traveling in a background material (see figure 2.1) with wavefront speed is $c$ (with $\frac{\omega}{|\mathbf{k}|}=c$ ). We introduce $c_{b}$ the relative speed of the wave in this material, such that $c=c_{b} c_{0}$, and denote $\varepsilon_{b}$ and $\mu_{b}$ the corresponding relative electromagnetic parameters. The permittivity and permeability of the background material are thus respectively given by $\varepsilon=\varepsilon_{b} \varepsilon_{0}$, $\mu=\mu_{b} \mu_{0}$. In the following, we consider that permittivity and permeability $\varepsilon_{b}$ and $\mu_{b}$ are scalar positive constants. We define a normalized wave vector:

$$
\hat{\mathbf{k}}=\frac{\mathbf{k}}{|\mathbf{k}|}={ }^{t}\left(\kappa_{x}, \kappa_{y}, \kappa_{z}\right)={ }^{t}(\sin \theta \cos \phi, \sin \theta \sin \phi, \cos \theta)
$$




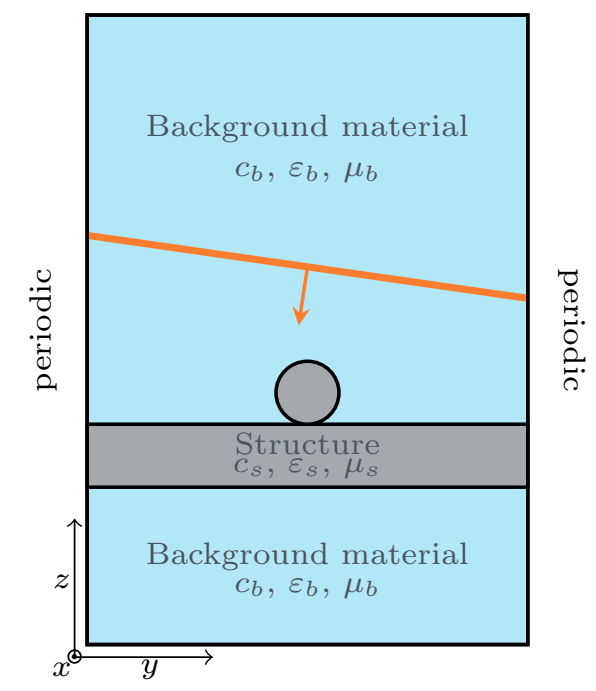

FIGURE 2.1. Oblique incidence configuration with a scattering structure embedded in a background material. An incident incoming oblique plane wave is imposed in the background material. It is reflected and diffracted by the scatterer (here, a sphere on a substrate). The domain is periodic in the $x$ and $y$ directions and unbounded in the $z$ direction.

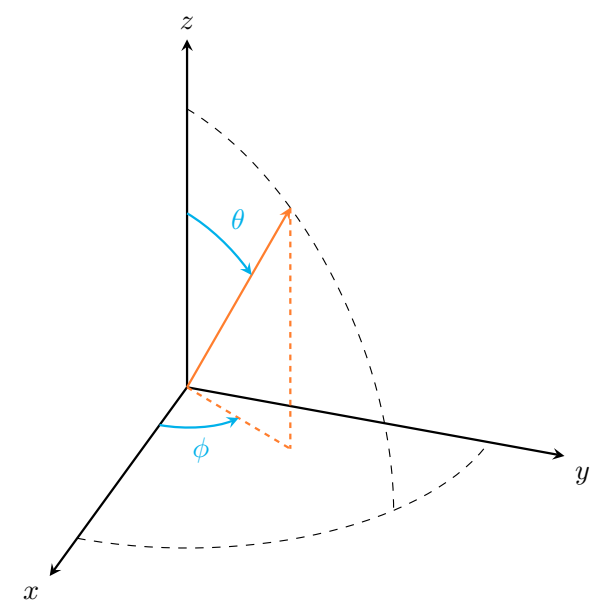

Figure 2.2. Definition of $(\theta, \phi)$ angles in the cartesian coordinate system.

where $|\mathbf{k}|=\frac{\omega}{c}$, and $(\theta, \phi)$ are defined as the classical spherical angles, see figure 2.2. The vector $\hat{\mathbf{k}}$ is decomposed in $\hat{\mathbf{k}}=\hat{\mathbf{k}}_{\|}+\hat{\mathbf{k}}_{\perp}$, where $\hat{\mathbf{k}}_{\perp}$ is oriented in the common direction of the periodicity planes, which is here taken to be $z$-axis for the sake of simplicity. Then:

$$
\hat{\mathbf{k}}_{\|}={ }^{t}\left(\kappa_{x}, \kappa_{y}, 0\right) \text { and } \hat{\mathbf{k}}_{\perp}={ }^{t}\left(0,0, \kappa_{z}\right) .
$$

We then consider a bi-periodic structure (in $x$ and $y$ directions, see figure 2.1) with electromagnetic positive constants $\varepsilon=\varepsilon_{0} \varepsilon_{s}$ and $\mu=\mu_{0} \mu_{s}$ ( $\varepsilon_{s}$ and $\mu_{s}$ being the relative parameters) embedded in the background material. The permittivity and permeability $\varepsilon_{s}$ and $\mu_{s}$ are scalar piecewise constants. We 
denote $c_{s}=\frac{1}{\sqrt{\varepsilon_{s} \mu_{s}}}$ the relative speed in the structure. Let us now recall the source-free frequencydomain Maxwell's equations in a media of electromagnetic constants $(\varepsilon, \mu)$ :

$$
\begin{aligned}
j \omega \varepsilon \mathbf{E}(\mathbf{r}, \omega) & =\nabla \times \mathbf{H}(\mathbf{r}, \omega), \\
j \omega \mu \mathbf{H}(\mathbf{r}, \omega) & =-\nabla \times \mathbf{E}(\mathbf{r}, \omega) .
\end{aligned}
$$

The field transform technique consists in formally considering the following transformation:

$$
\begin{aligned}
\mathbf{P}(\mathbf{r}, \omega) & =\mathbf{E}(\mathbf{r}, \omega) \exp \left(j \mathbf{k}_{\|} \cdot \mathbf{r}\right), \\
\mathbf{S}(\mathbf{r}, \omega) & =\mathbf{H}(\mathbf{r}, \omega) \exp \left(j \mathbf{k}_{\|} \cdot \mathbf{r}\right) .
\end{aligned}
$$

This transformation generates the transformed fields $\mathrm{P}$ and $\mathrm{S}$ that are not anymore affected by the tilting of oblique incidence, and are therefore periodic in $x$ and $y$ directions.

Applying transformation (2.2) to system (2.1), a few lines of calculations along with the identity $\nabla \times(\psi \mathbf{V})=(\nabla \psi) \times \mathbf{V}+\psi(\nabla \times \mathbf{V})$ lead to:

$$
\begin{aligned}
& j \omega \varepsilon \mathbf{P}(\mathbf{r}, \omega)=\nabla \times \mathbf{S}(\mathbf{r}, \omega)-j \mathbf{k}_{\|} \times \mathbf{S}(\mathbf{r}, \omega), \\
& j \omega \mu \mathbf{S}(\mathbf{r}, \omega)=-\nabla \times \mathbf{P}(\mathbf{r}, \omega)+j \mathbf{k}_{\|} \times \mathbf{P}(\mathbf{r}, \omega) .
\end{aligned}
$$

Formally transforming back to time-domain with the identity $j \omega \leftrightarrow \frac{\partial}{\partial t}$ and recalling that $\mathbf{k}_{\|}=\hat{\mathbf{k}}_{\|}|\mathbf{k}|$ yields the following system in the time dependent equations:

$$
\begin{aligned}
& \varepsilon \frac{\partial \mathbf{P}}{\partial t}(\mathbf{r}, t)+\frac{\hat{\mathbf{k}}_{\|}}{c_{0} c_{b}} \times \frac{\partial \mathbf{S}}{\partial t}(\mathbf{r}, t)=\nabla \times \mathbf{S}(\mathbf{r}, t), \\
& \mu \frac{\partial \mathbf{S}}{\partial t}(\mathbf{r}, t)-\frac{\hat{\mathbf{k}}_{\|}}{c_{0} c_{b}} \times \frac{\partial \mathbf{P}}{\partial t}(\mathbf{r}, t)=-\nabla \times \mathbf{P}(\mathbf{r}, t) .
\end{aligned}
$$

Finally, for the sake of simplicity, we classically normalize the latter system following [18] to obtain:

$$
\begin{aligned}
& \varepsilon_{r} \frac{\partial \mathbf{P}}{\partial t}(\mathbf{r}, t)+\frac{\hat{\mathbf{k}}_{\|}}{c_{b}} \times \frac{\partial \mathbf{S}}{\partial t}(\mathbf{r}, t)=\nabla \times \mathbf{S}(\mathbf{r}, t), \\
& \mu_{r} \frac{\partial \mathbf{S}}{\partial t}(\mathbf{r}, t)-\frac{\hat{\mathbf{k}}_{\|}}{c_{b}} \times \frac{\partial \mathbf{P}}{\partial t}(\mathbf{r}, t)=-\nabla \times \mathbf{P}(\mathbf{r}, t) .
\end{aligned}
$$

with $\varepsilon_{r}=\varepsilon_{s}, \mu_{r}=\mu_{s}$ in the structure and $\varepsilon_{r}=\varepsilon_{b}, \mu_{r}=\mu_{b}$ in the background material. Forming the vector $\mathbf{U}={ }^{t}(\mathbf{P}, \mathbf{S})$, one can recast system (2.3) under a more general form:

$$
\mathbb{Q} \frac{\partial \mathbf{U}}{\partial t}+\mathbb{A} \frac{\partial \mathbf{U}}{\partial x}+\mathbb{B} \frac{\partial \mathbf{U}}{\partial y}+\mathbb{C} \frac{\partial \mathbf{U}}{\partial z}=0
$$

where $\mathbb{A}, \mathbb{B}, \mathbb{C}$ are the usual coefficient matrices (see definition in [15]), and $\mathbb{Q}$ is defined as:

$$
\mathbb{Q}=\frac{1}{c_{b}}\left[\begin{array}{cccccc}
c_{b} \varepsilon_{r} & 0 & 0 & 0 & 0 & \kappa_{y} \\
0 & c_{b} \varepsilon_{r} & 0 & 0 & 0 & -\kappa_{x} \\
0 & 0 & c_{b} \varepsilon_{r} & -\kappa_{y} & \kappa_{x} & 0 \\
0 & 0 & -\kappa_{y} & c_{b} \mu_{r} & 0 & 0 \\
0 & 0 & \kappa_{x} & 0 & c_{b} \mu_{r} & 0 \\
\kappa_{y} & -\kappa_{x} & 0 & 0 & 0 & c_{b} \mu_{r}
\end{array}\right] .
$$

Note that system (2.3) both depends (i) on the local material, through $\varepsilon_{r}$ and $\mu_{r}$, and (ii) on the background material, through its relative speed of light $c_{b}$. The background material is the material of the infinite surrounding, where the incident field is imposed. Its introduction in the transformation allows to tackle cases where the considered structure is not embedded in vacuum. The latter system can be analyzed on the structure (i.e. in the part of the domain occupied by the structure, $r=s$ ) using an eigenvalue study. 
TABLE 2.1. Characteristic speeds for system (2.4), with $\bar{c}=\frac{c_{b}}{c_{s}}$.

\begin{tabular}{cc}
\hline$\lambda_{i}$ & Multiplicity \\
\hline$\frac{\kappa_{x}+\sqrt{\bar{c}^{2}-\kappa_{y}^{2}}}{\bar{c}^{2}-\kappa_{x}^{2}-\kappa_{y}^{2}} c_{b}$ & 2 \\
\hline$\frac{\kappa_{x}-\sqrt{\bar{c}^{2}-\kappa_{y}^{2}}}{\bar{c}^{2}-\kappa_{x}^{2}-\kappa_{y}^{2}} c_{b}$ & 2 \\
\hline$\frac{\kappa_{y}+\sqrt{\bar{c}^{2}-\kappa_{x}^{2}}}{\bar{c}^{2}-\kappa_{x}^{2}-\kappa_{y}^{2}} c_{b}$ & 2 \\
\hline$\frac{\kappa_{y}-\sqrt{\bar{c}^{2}-\kappa_{x}^{2}}}{\bar{c}^{2}-\kappa_{x}^{2}-\kappa_{y}^{2}} c_{b}$ & 2 \\
\hline$\frac{c_{b}}{\sqrt{\bar{c}^{2}-\kappa_{x}^{2}-\kappa_{y}^{2}}}$ & 2 \\
\hline$\frac{-c_{b}}{\sqrt{\bar{c}^{2}-\kappa_{x}^{2}-\kappa_{y}^{2}}}$ & 2 \\
\hline 0 & 6 \\
\hline
\end{tabular}

\subsection{Characteristics analysis}

For a hyperbolic system, we can follow the method of characteristics [15], use the characteristic variables (which are not detailed here) and conclude that they will travel along trajectories which slopes are the eigenvalues of $\mathbb{Q}^{-1} \mathbb{A}, \mathbb{Q}^{-1} \mathbb{B}$ and $\mathbb{Q}^{-1} \mathbb{C}$. These eigenvalues are shown in table 2.1. They play a central role when considering space time approximation of the system and using an explicit time integration scheme. In this subsection, we thus also exploit the information given by the eigenvalues to have an insight on the possible stability of a future explicit time integration scheme (through a fictive time-step and CFL type condition). Let us now analyze the behavior of these eigenvalues in a few basic cases. For the sake of simplicity, in this section, we restrict ourselves to non-magnetic materials $\left(\mu_{b}=\mu_{s}=1\right)$.

\subsection{1. $\phi=0, \bar{c}=1$}

Having $\phi=0$ (i.e. the wave will propagate parallel to the $\{y=0\}$ plane) yields $\kappa_{x}=\sin \theta$ and $\kappa_{y}=0$. Additionally, $\bar{c}=1$ indicates that we are looking at the behavior of the eigenvalues of system (2.3) in the background material. In this case, their expressions reduce to:

$$
\begin{aligned}
\lambda_{x^{+}, x^{-}} & =\frac{ \pm c_{b}}{1 \mp \sin \theta}, \\
\lambda_{y^{+}, y^{-}}(\theta) & =\frac{ \pm c_{b}}{\sqrt{1-\sin ^{2} \theta}}, \\
\lambda_{z^{+}, z^{-}} & (\theta)=\lambda_{y^{+}, y^{-}} .
\end{aligned}
$$

For $\theta=0^{\circ}$, one recovers the regular eigenvalues $\lambda= \pm c_{b}$ (which is true even for $\phi \neq 0$ ). The latter expressions show that, in vacuum, the speed of some characteristic variables of the transformed system (2.3) is superior to that of light as soon as $\theta>0^{\circ}$. This fact is coherent with the reduction of the largest stable time-step with increasing $\theta$ noted in [11], and with our own theoretical predictions and numerical experiments that will be presented in next sections. As shown in figure 2.3, the most constraining speed in terms of CFL value is $\lambda_{x^{+}}(\theta)$, meaning that the maximal stable time-step will be expected to decrease as $\frac{1}{\lambda_{x^{+}}(\theta)}$. 


\section{Simulating 3D Periodic StruCtures at oblique incidences}

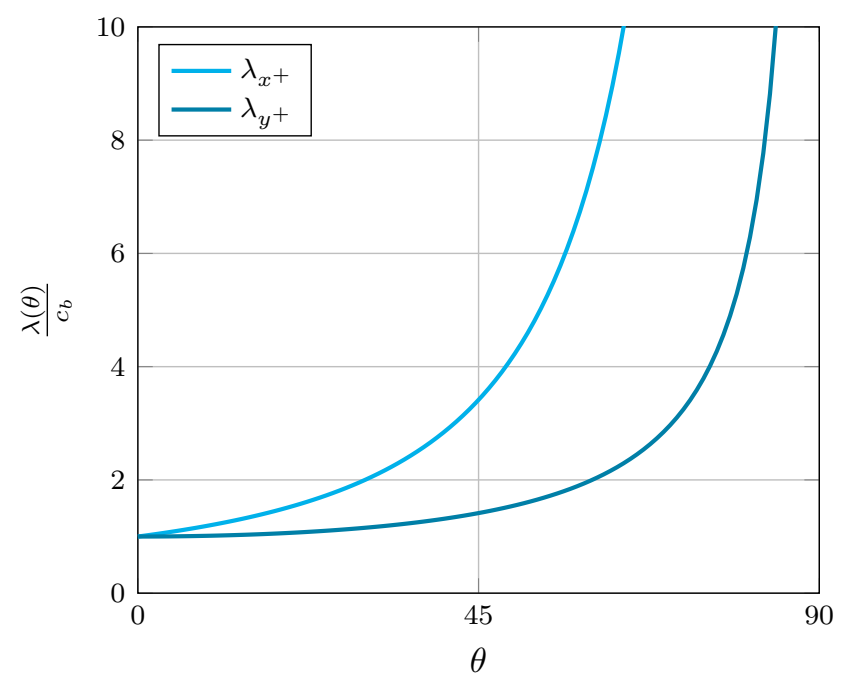

FIGURE 2.3. Normalized characteristic speeds of system (2.4) for $\phi=0$ and $\bar{c}=1$, with respect to the angle $\theta$ in degree.

\subsection{2. $\phi=0, \quad \bar{c} \neq 1$}

Having $\bar{c} \neq 1$ means that we are considering the solutions of system (2.3) in a material that is not the background material. The eigenvalues then have the following expressions:

$$
\begin{aligned}
\lambda_{x^{+}, x^{-}}(\theta) & =\frac{ \pm c_{b}}{\bar{c} \mp \sin \theta}, \\
\lambda_{y^{+}, y^{-}}(\theta) & =\frac{ \pm c_{b}}{\sqrt{\bar{c}^{2}-\sin ^{2} \theta}}, \\
\lambda_{z^{+}, z^{-}}(\theta) & =\lambda_{y^{+}, y^{-}},
\end{aligned}
$$

The speed of the characteristic variables is still superior to that of the background material. However, two cases now need to be distinguished:

(1) $\bar{c}>1$, i.e. $\varepsilon_{s}>\varepsilon_{b}$ (for example, a dielectric slab embedded in vacuum). All values of $\theta$ in the range $\left[0^{\circ}, 90^{\circ}[\right.$ are acceptable;

(2) $\bar{c}<1$, i.e. $\varepsilon_{s}<\varepsilon_{b}$ (for example, a vacuum slab between two dielectric half-spaces). In this case, $\lambda_{x^{+}}(\theta)$ is singular for a critical value $\theta_{c}$ :

$$
\theta_{c}(\bar{c})=\sin ^{-1} \bar{c},
$$

while $\lambda_{y^{+}}(\theta)$ and $\lambda_{y^{-}}(\theta)$ become imaginary with opposite signs for $\theta>\theta_{c}$. A plot of $\theta_{c}$ as a function of $\bar{c}$ is given in figure 2.4. Following Snell's law, it should be noted that $\theta_{c}$ is nothing else than the angle of total internal reflection, for which all the light will end up travelling along the materials interface. A direct consequence is that we do not expect to find a stable time step for the discretization of system (2.3) for $\theta \geq \theta_{c}$ if $\bar{c}<1$. This will be confirmed with the rest of our study.

\subsubsection{General case}

For any $\phi$ values, we recover the critical angle. Indeed, to have a well defined positive square root in the eigenvalue expression (which will allow for selecting a possible stable time-step in the discrete 


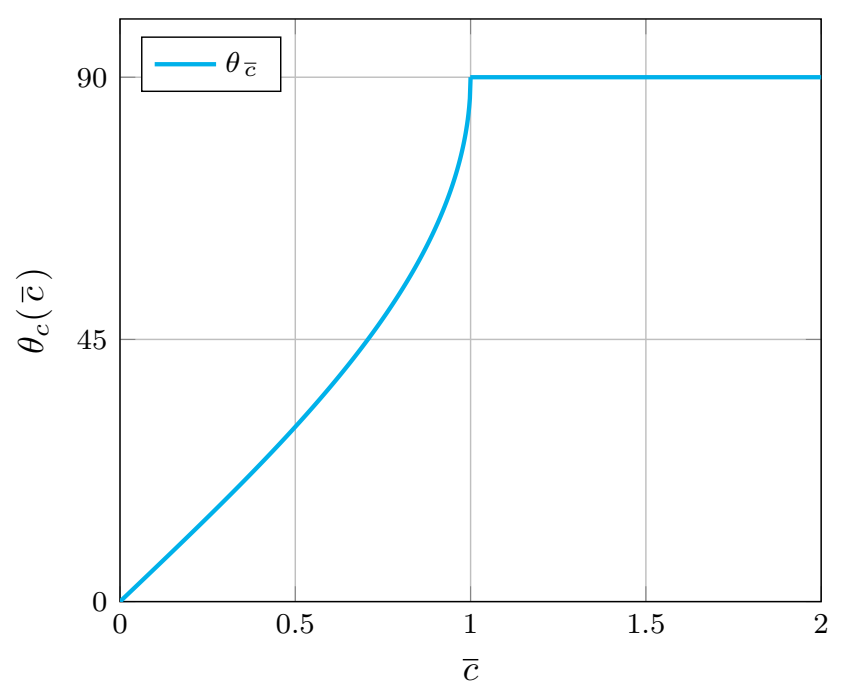

Figure 2.4. Plot of the critical value $\theta_{c}$ as a function of $\bar{c}$ in the range $[0,1[$. For $\bar{c}>1, \theta$ can take all values in $\left[0,90^{\circ}\right.$.

setting), we have to impose that:

$$
\bar{c}^{2}-\kappa_{x}^{2}-\kappa_{y}^{2}>0
$$

which implies:

$$
\sin ^{2}(\theta)<\bar{c}^{2} .
$$

Imposing this condition suffices to have both $\bar{c}^{2}-\kappa_{y}^{2} \geq 0$ and $\bar{c}^{2}-\kappa_{x}^{2} \geq 0$.

\subsection{Energy principle and well-posedness}

In this section, we investigate the energy of the system and give a well defined mathematical setting. We truncate the physical domain in the $z$ direction and denote the resulting open bounded domain by $\Omega \subset \mathbb{R}^{3}$ (see figure 2.5). The boundary of $\Omega, \Gamma$, thus consists of several parts: $\Gamma_{\text {ext }}$ the exterior artificial boundary and $\Gamma_{P}$, the periodic boundary. On $\Gamma_{\text {ext }}$ using a scattered field formulation, will be either imposed absorbing boundary conditions or a layer of PML's (see next section). The domain $\Omega$ itself consists in the background material $\Omega_{b}$ (with physical parameters $\varepsilon_{r}=\varepsilon_{b}$ and $\mu_{r}=\mu_{b}$ ) and the structure $\Omega_{s}$ (with physical parameters $\varepsilon_{r}=\varepsilon_{s}$ and $\mu_{r}=\mu_{s}$ ).

We denote by $\mathbf{L}^{2}(\Omega)$, the space of vectorial functions that are real valued and square integrable on $\Omega, H(\operatorname{curl}, \Omega)$ the space of $\mathbf{L}^{2}(\Omega)$ fields with square integrable curl, $\langle\cdot, \cdot\rangle_{\mathcal{D}}$ and $\|\cdot\|_{\mathcal{D}}$ respectively the $L^{2}$ scalar product and $L^{2}$ norm on a given set $\mathcal{D}$. Finally we define $\mathcal{H}=\mathbf{L}^{2}(\Omega) \times \mathbf{L}^{2}(\Omega)$.

We consider the equations on a fixed time interval $[0, T], T>0$. To summarize, we consider the following system of equations, for all $t \in[0, T]$ :

$$
\begin{aligned}
& \varepsilon_{r} \frac{\partial \mathbf{P}}{\partial t}(\cdot, t)+\frac{\hat{\mathbf{k}}_{\|}}{c_{b}} \times \frac{\partial \mathbf{S}}{\partial t}(\cdot, t)=\nabla \times \mathbf{S}(\cdot, t), \text { on } \Omega, \\
& \mu_{r} \frac{\partial \mathbf{S}}{\partial t}(\cdot, t)-\frac{\hat{\mathbf{k}}_{\|}}{c_{b}} \times \frac{\partial \mathbf{P}}{\partial t}(\cdot, t)=-\nabla \times \mathbf{P}(\cdot, t), \text { on } \Omega .
\end{aligned}
$$

These equations are supplemented with initial and boundary conditions. For the latter, we impose $\mathrm{PBC}$ on the lateral boundaries of the domain (see figure 2.5). The outer boundaries are not specified right now to be able to envisage several truncation alternatives. 


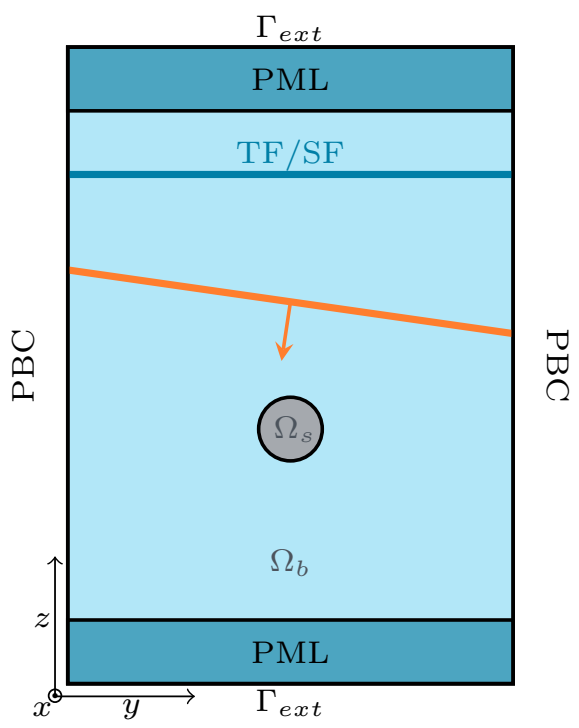

Figure 2.5. Notations. The domain is periodic in the lateral directions, truncated on $\Gamma_{\text {ext }}$. The incident plane wave is imposed at the Total-Field/Scattered-Field (TF/SF) interface, and is oriented toward the bottom of the domain. The artificial truncation can be handled via e.g. a layer of Perfectly Matched Layers (PML's), see later

We begin our study by an a priori analysis. In other words, we suppose that there exists a unique smooth solution $(\mathbf{P}, \mathbf{S})$ and provide an energy study in the next two propositions. This study will be the basis for a discussion on well-posedness.

We define $V$ a subspace of $H(\operatorname{curl}, \Omega)$ for which an integration by part formula

$$
\forall(f, g) \in V \times V,\langle\nabla \times f, g\rangle-\langle f, \nabla \times g\rangle=b_{\partial \Omega}(f, g)
$$

holds, with $b_{\partial \Omega}$ a bilinear boundary term such that $b_{\partial \Omega}=b_{\Gamma_{e x t}}+b_{\Gamma_{P}}$. If $V$ is a space of fields with well defined $L^{2}$ tangential traces, one has $b_{\partial \Omega}=\langle f \times n, g\rangle_{\partial \Omega}$ which can be understood in the usual $L^{2}$ sense. If $V$ consists of less regular fields (e.g. only $H(\operatorname{curl}, \Omega)$ ), using e.g. the general theory developed in $\left[1\right.$, Chapter 3], one can give a rigorous sense to $b_{\partial \Omega}$.

Proposition 2.1. Assume that $(\boldsymbol{P}, \boldsymbol{S}) \in \mathcal{C}^{1}(0, T, V)^{2}$ and that $(\boldsymbol{P}, \boldsymbol{S})$ verify $(2.6)$. If for all $t \in[0, T]$,

$$
\mathcal{E}(t):=\frac{1}{2}\left\|\sqrt{\varepsilon_{r}} \boldsymbol{P}(t, \cdot)\right\|_{\Omega}^{2}+\frac{1}{2}\left\|\sqrt{\mu_{r}} \boldsymbol{S}(t, \cdot)\right\|_{\Omega}^{2}+\left\langle\frac{\hat{\boldsymbol{k}}_{\|}}{c_{b}} \times \boldsymbol{S}(t, \cdot), \boldsymbol{P}(t, \cdot)\right\rangle_{\Omega} .
$$

Then $\mathcal{E}(t)=\mathcal{E}(0)+\int_{0}^{t} b_{\Gamma_{e x t}}(\boldsymbol{S}, \boldsymbol{P})$.

Proof. We take the $L^{2}$-scalar product of $(2.6)$ with test function $(\mathbf{S}, \mathbf{P})$. We obtain:

$$
\begin{aligned}
& \left\langle\varepsilon_{r} \frac{\partial \mathbf{P}}{\partial t}, \mathbf{P}\right\rangle_{\Omega}+\left\langle\frac{\hat{\mathbf{k}}_{\|}}{c_{b}} \times \frac{\partial \mathbf{S}}{\partial t}, \mathbf{P}\right\rangle_{\Omega}=\langle\nabla \times \mathbf{S}, \mathbf{P}\rangle_{\Omega}, \\
& \left\langle\mu_{r} \frac{\partial \mathbf{S}}{\partial t}, \mathbf{S}\right\rangle_{\Omega}-\left\langle\frac{\hat{\mathbf{k}}_{\|}}{c_{b}} \times \frac{\partial \mathbf{P}}{\partial t}, \mathbf{S}\right\rangle_{\Omega}=-\langle\nabla \times \mathbf{P}, \mathbf{S}\rangle_{\Omega},
\end{aligned}
$$


which is equivalent to:

$$
\begin{aligned}
& \frac{1}{2} \frac{\partial}{\partial t}\left\langle\varepsilon_{r} \mathbf{P}, \mathbf{P}\right\rangle_{\Omega}+\left\langle\frac{\hat{\mathbf{k}}_{\|}}{c_{b}} \times \frac{\partial \mathbf{S}}{\partial t}, \mathbf{P}\right\rangle_{\Omega}=\langle\nabla \times \mathbf{S}, \mathbf{P}\rangle_{\Omega}, \\
& \frac{1}{2} \frac{\partial}{\partial t}\left\langle\mu_{r} \mathbf{S}, \mathbf{S}\right\rangle_{\Omega}-\left\langle\frac{\hat{\mathbf{k}}_{\|}}{c_{b}} \times \frac{\partial \mathbf{P}}{\partial t}, \mathbf{S}\right\rangle_{\Omega}=-\langle\nabla \times \mathbf{P}, \mathbf{S}\rangle_{\Omega} .
\end{aligned}
$$

We can make use of the integration by part formula (2.7) to obtain:

$$
\begin{aligned}
& \frac{1}{2} \frac{\partial}{\partial t}\left\langle\varepsilon_{r} \mathbf{P}, \mathbf{P}\right\rangle_{\Omega}+\left\langle\frac{\hat{\mathbf{k}}_{\|}}{c_{b}} \times \frac{\partial \mathbf{S}}{\partial t}, \mathbf{P}\right\rangle_{\Omega}=\langle\mathbf{S}, \nabla \times \mathbf{P}\rangle_{\Omega}+b_{\partial \Omega}(\mathbf{S}, \mathbf{P}), \\
& \frac{1}{2} \frac{\partial}{\partial t}\left\langle\mu_{r} \mathbf{S}, \mathbf{S}\right\rangle_{\Omega}-\left\langle\frac{\hat{\mathbf{k}}_{\|}}{c_{b}} \times \frac{\partial \mathbf{P}}{\partial t}, \mathbf{S}\right\rangle_{\Omega}=-\langle\nabla \times \mathbf{P}, \mathbf{S}\rangle_{\Omega} .
\end{aligned}
$$

One distinguishes contributions on $\Gamma_{e x t}$ and $\Gamma_{P}$ :

$$
b_{\partial \Omega}(\mathbf{S}, \mathbf{P})=b_{\Gamma_{P}}(\mathbf{S}, \mathbf{P})+b_{\Gamma_{e x t}}(\mathbf{S}, \mathbf{P}) .
$$

Using the periodic boundary conditions on $\Gamma_{P}$, we deduce that $b_{\Gamma_{P}}(\mathbf{S}, \mathbf{P})=0$. Summing the two equations:

$$
\frac{1}{2} \frac{\partial}{\partial t}\left\langle\varepsilon_{r} \mathbf{P}, \mathbf{P}\right\rangle_{\Omega}+\frac{1}{2} \frac{\partial}{\partial t}\left\langle\mu_{r} \mathbf{S}, \mathbf{S}\right\rangle_{\Omega}+\left\langle\frac{\hat{\mathbf{k}}_{\|}}{c_{b}} \times \frac{\partial \mathbf{S}}{\partial t}, \mathbf{P}\right\rangle_{\Omega}-\left\langle\frac{\hat{\mathbf{k}}_{\|}}{c_{b}} \times \frac{\partial \mathbf{P}}{\partial t}, \mathbf{S}\right\rangle_{\Omega}=b_{\Gamma_{e x t}}(\mathbf{S}, \mathbf{P})
$$

Using the properties of the mixed product, one has:

$$
\frac{1}{2} \frac{\partial}{\partial t}\left\langle\varepsilon_{r} \mathbf{P}, \mathbf{P}\right\rangle_{\Omega}+\frac{1}{2} \frac{\partial}{\partial t}\left\langle\mu_{r} \mathbf{S}, \mathbf{S}\right\rangle_{\Omega}+\left\langle\frac{\hat{\mathbf{k}}_{\|}}{c_{b}} \times \frac{\partial \mathbf{S}}{\partial t}, \mathbf{P}\right\rangle_{\Omega}+\left\langle\frac{\hat{\mathbf{k}}_{\|}}{c_{b}} \times \mathbf{S}, \frac{\partial \mathbf{P}}{\partial t}\right\rangle_{\Omega}=b_{\Gamma_{e x t}}(\mathbf{S}, \mathbf{P}) .
$$

Thus,

$$
\frac{1}{2} \frac{\partial}{\partial t}\left\langle\varepsilon_{r} \mathbf{P}, \mathbf{P}\right\rangle_{\Omega}+\frac{1}{2} \frac{\partial}{\partial t}\left\langle\mu_{r} \mathbf{S}, \mathbf{S}\right\rangle_{\Omega}+\frac{\partial}{\partial t}\left\langle\frac{\hat{\mathbf{k}}_{\|}}{c_{b}} \times \mathbf{S}, \mathbf{P}\right\rangle_{\Omega}=b_{\Gamma_{e x t}}(\mathbf{S}, \mathbf{P}),
$$

and the result follows.

We now prove that the energy $\mathcal{E}$ is positive under some assumption on $\hat{\mathbf{k}}$ and apply this result in the special cases considered in this paper.

Proposition 2.2. Under the hypotheses and notations of Proposition 2.1, one has for all $t \in[0, T]$ :

$$
\begin{aligned}
\mathcal{E}(t) & \geq \frac{1}{2}\left(1-\frac{\left\|\hat{\boldsymbol{k}}_{\|}\right\|}{\bar{c}}\right)\left\|\sqrt{\varepsilon_{r}} \boldsymbol{P}(t, \cdot)\right\|_{\Omega_{s}}^{2}+\frac{1}{2}\left(1-\frac{\left\|\hat{\boldsymbol{k}}_{\|}\right\|}{\bar{c}}\right)\left\|\sqrt{\mu_{r}} \boldsymbol{S}(t, \cdot)\right\|_{\Omega_{s}}^{2} \\
& +\frac{1}{2}\left(1-\left\|\hat{\boldsymbol{k}}_{\|}\right\|\right)\left\|\sqrt{\varepsilon_{r}} \boldsymbol{P}(t, \cdot)\right\|_{\Omega_{b}}^{2}+\frac{1}{2}\left(1-\left\|\hat{\boldsymbol{k}}_{\|}\right\|\right)\left\|\sqrt{\mu_{r}} \boldsymbol{S}(t, \cdot)\right\|_{\Omega_{b}}^{2} .
\end{aligned}
$$

If one supposes that $\min (\bar{c}, 1)>\left\|\hat{\boldsymbol{k}}_{\|}\right\|$, then $\mathcal{E}(t)$ is positive quadratic form for $t \in[0, T]$. Furthermore, if the exterior boundary conditions are chosen such that there exists $M \geq 0$ such that for all $t \in[0, T]$, $\int_{0}^{t} b_{\Gamma_{e x t}}(\boldsymbol{S}, \boldsymbol{P}) \leq M$, then:

$$
\mathcal{E}(t) \leq \mathcal{E}(0)+M
$$

i.e. the positive energy is bounded. 
Proof. One has:

$$
\begin{aligned}
\left|\left\langle\frac{\hat{\mathbf{k}}_{\|}}{c_{b}} \times \mathbf{S}, \mathbf{P}\right\rangle_{\Omega_{s}}\right| & \leq \frac{\left\|\hat{\mathbf{k}}_{\|}\right\|}{c_{b} \sqrt{\varepsilon_{s}} \sqrt{\mu_{s}}}\left\|\sqrt{\mu_{r}} \mathbf{S}\right\|_{\Omega_{s}}\left\|\sqrt{\varepsilon_{r}} \mathbf{P}\right\|_{\Omega_{s}}, \\
& \leq \frac{\left\|\hat{\mathbf{k}}_{\|}\right\|}{\bar{c}}\left\|\sqrt{\mu_{r}} \mathbf{S}\right\|_{\Omega_{s}}\left\|\sqrt{\varepsilon_{r}} \mathbf{P}\right\|_{\Omega_{s}} .
\end{aligned}
$$

Using Young's inequality, we obtain

This implies that:

$$
\left|\left\langle\frac{\hat{\mathbf{k}}_{\|}}{c_{b}} \times \mathbf{S}, \mathbf{P}\right\rangle_{\Omega_{s}}\right| \leq \frac{1}{2} \frac{\left\|\hat{\mathbf{k}}_{\|}\right\|}{\bar{c}}\left\|\sqrt{\varepsilon_{r}} \mathbf{P}\right\|_{\Omega_{s}}^{2}+\frac{1}{2} \frac{\left\|\hat{\mathbf{k}}_{\|}\right\|}{\bar{c}}\left\|\sqrt{\mu_{r}} \mathbf{S}\right\|_{\Omega_{s}}^{2} .
$$

$$
\left\langle\frac{\hat{\mathbf{k}}_{\|}}{c_{b}} \times \mathbf{S}, \mathbf{P}\right\rangle_{\Omega_{s}} \geq-\left(\frac{1}{2} \frac{\left\|\hat{\mathbf{k}}_{\|}\right\|}{\bar{c}}\left\|\sqrt{\varepsilon_{r}} \mathbf{P}\right\|_{\Omega_{s}}^{2}+\frac{1}{2} \frac{\left\|\hat{\mathbf{k}}_{\|}\right\|}{\bar{c}}\left\|\sqrt{\mu_{r}} \mathbf{S}\right\|_{\Omega_{s}}^{2}\right) .
$$

In a similar manner, we obtain

$$
\left\langle\frac{\hat{\mathbf{k}}_{\|}}{c_{b}} \times \mathbf{S}, \mathbf{P}\right\rangle_{\Omega_{b}} \geq-\left(\frac{1}{2}\left\|\hat{\mathbf{k}}_{\|}\right\|\left\|\sqrt{\varepsilon_{r}} \mathbf{P}\right\|_{\Omega_{b}}^{2}+\frac{1}{2}\left\|\hat{\mathbf{k}}_{\|}\right\|\left\|\sqrt{\mu_{r}} \mathbf{S}\right\|_{\Omega_{b}}^{2}\right) .
$$

Combining these last two estimates with the definition of $\mathcal{E}$, we obtain (2.8). The conclusions then follow from (2.8) and Proposition 2.1.

Remark 2.3. If one considers absorbing boundary conditions (first order Silver-Müller) or perfect electric conductor boundary condition on $\Gamma_{e x t}$, then we expect $b_{\Gamma_{e x t}}(\mathbf{S}, \mathbf{P}) \leq 0$. In this case, the energy is decreasing.

Remark 2.4. We recover the stability condition inferred in the characteristic analysis through the condition $\left\|\hat{\mathbf{k}}_{\|}\right\| \leq \bar{c}$.

Remark 2.5. We would like to point out that this study naturally extends to classical dispersion models such as the Drude model or even generalized dispersion laws that are commonly used in nanophotonics. Indeed, these models consist only in adding auxiliary ordinary differential equations (see [8] for details on the models) that are linearly coupled to Maxwell equations via a source current. For readability, we do not detail this extension here, since it is straightforward. We refer the reader to the discussion on this topic in section 3.5).

This first a priori study set the basis for possible results of well posedness. To this end, we introduce the following bilinear form on $\mathcal{H} \times \mathcal{H}$ :

$a_{\mathbb{Q}}:\left(u=\left(u_{1}, u_{2}\right), v=\left(v_{1}, v_{2}\right)\right) \mapsto \frac{1}{2}\left(\left\langle\varepsilon_{r} u_{1}, v_{1}\right\rangle_{\Omega}+\left\langle\mu_{r} u_{2}, v_{2}\right\rangle_{\Omega}+\left\langle\frac{\hat{\mathbf{k}}_{\|}}{c_{b}} \times u_{1}, v_{2}\right\rangle_{\Omega}+\left\langle\frac{\hat{\mathbf{k}}_{\|}}{c_{b}} \times v_{1}, u_{2}\right\rangle_{\Omega}\right)$.

This bilinear form is symmetric. Furthermore, if $\min (\bar{c}, 1)>\left\|\hat{\mathbf{k}}_{\|}\right\|, a_{\mathbb{Q}}$ is positive definite. Indeed, first for all $u=\left(u_{1}, u_{2}\right) \in \mathcal{H}$ on has

$$
a_{\mathbb{Q}}(u, u)=\frac{1}{2}\left(\left\langle\varepsilon_{r} u_{1}, u_{1}\right\rangle_{\Omega}+\left\langle\mu_{r} u_{2}, u_{2}\right\rangle_{\Omega}+2\left\langle\frac{\hat{\mathbf{k}}_{\|}}{c_{b}} \times u_{1}, u_{2}\right\rangle_{\Omega}\right) .
$$

Following the proof of Proposition 2.2, we have,

$$
a_{\mathbb{Q}}(u, u) \geq \frac{1}{2} \min \left(1-\frac{\left\|\hat{\mathbf{k}}_{\|}\right\|}{\bar{c}}, 1-\left\|\hat{\mathbf{k}}_{\|}\right\|\right)\left(\left\|\sqrt{\varepsilon_{r}} u_{1}\right\|_{\Omega}^{2}+\left\|\sqrt{\mu_{r}} u_{2}\right\|_{\Omega}^{2}\right) .
$$


This yields that $a_{\mathbb{Q}}$ is positive definite and thus is a scalar product, if $\min (\bar{c}, 1)>\left\|\hat{\mathbf{k}}_{\|}\right\|$. We define $\|\cdot\|_{\mathbb{Q}}$, the associated norm. This norm is equivalent to the canonical $\mathcal{H}$ norm. Thus $\left(\mathcal{H}, a_{\mathbb{Q}}\right)$ is a Hilbert space.

Let us now define the unbounded Maxwell operator $\mathcal{A}_{M}$ with domain $\mathcal{D}\left(\mathcal{A}_{M}\right)$ as follows: for all $u=\left(u_{1}, u_{2}\right) \in \mathcal{D}\left(\mathcal{A}_{M}\right), \mathcal{A}_{M}(u)$ is given by

$$
\mathcal{A}_{M}(u)=\left(\begin{array}{l}
\nabla \times u_{2} \\
-\nabla \times u_{1}
\end{array}\right) .
$$

Remark 2.6. At this step, we do not explicitly specify the domain $\mathcal{D}\left(\mathcal{A}_{M}\right)$ to include more generality in the results.

Furthermore, we define $\mathcal{Q}$ the linear continuous operator on $\mathcal{H}$, defined for all $u=\left(u_{1}, u_{2}\right)$ by $\mathcal{Q}(u)=\mathbb{Q}^{-1} u$.

We can rewrite the set of equations (2.6) as a Cauchy problem

$$
\left\{\begin{array}{l}
\partial_{t} u=\mathcal{Q} \circ \mathcal{A}_{M}(u), \\
u(0)=u_{0},
\end{array}\right.
$$

with $u_{0} \in \mathcal{D}\left(\mathcal{A}_{M}\right)$.

Direct computations lead to the following.

Proposition 2.7. One has for all $u, v$ in $\mathcal{D}\left(\mathcal{A}_{M}\right)$

$$
a_{\mathbb{Q}}\left(\mathcal{Q} \circ \mathcal{A}_{M}(u), v\right)=\left\langle\mathcal{A}_{M}(u), v\right\rangle_{\Omega},
$$

This proposition implies that well-posedness study of (2.11) is closely related to the study of wellposedness of Maxwell's equation. Indeed, from this proposition, we deduce in particular that if $\mathcal{A}_{M}$ has an adjoint $\mathcal{A}_{M}^{*}$ (with domain $\left.\mathcal{D}\left(\mathcal{A}_{M}\right)\right)$ in $(\mathcal{H},\langle\cdot, \cdot\rangle)$, then so does $\mathcal{Q} \circ \mathcal{A}_{M}$ in $\left(\mathcal{H}, a_{\mathbb{Q}}\right)$ and this adjoint is $\mathcal{Q} \circ \mathcal{A}_{M}^{*}$. Furthermore, if $\mathcal{A}_{M}$ (resp. $\mathcal{A}_{M}^{*}$ ) is monotone in $(\mathcal{H},\langle\cdot, \cdot\rangle)$, so is $\mathcal{Q} \circ \mathcal{A}_{M}$ (resp. $\left.\left(\mathcal{Q} \circ \mathcal{A}_{M}\right)^{*}\right)$ in $\left(\mathcal{H}, a_{\mathbb{Q}}\right)$. The same holds for skew-adjointness property. By specifying the domain $\mathcal{D}\left(\mathcal{A}_{M}\right)$, wellposedness results can therefore be obtained from results of well-posedness for the classical Maxwell case (i.e. with unbounded operator $\mathcal{A}_{M}$, with domain $\mathcal{D}\left(\mathcal{A}_{M}\right)$ ) using e.g. theorems as in $[1, \S 4.3$, p. 162]. As an example, considering periodic condition on $\Gamma_{P}$ and perfect electric conducting boundary condition $\left(u_{1} \times n=0\right)$ on $\Gamma_{\text {ext }}$ lead to skew adjointness of the operator, and Stone theorem (see e.g. [1, p. 164]) help to conclude. In other words, under suitable assumptions, one would be able to address well-posedness of $(2.11)$.

\section{The DGTD method}

We now turn to the numerical approximation. The discretization framework is based on a DGTD scheme. Since the type scheme that we use is well described in a significant number of references (among them see e.g. [11] in the context of PBC, [18, 8] in a nanophotonics context), we choose to give the reader a brief presentation of it and refer to the above mentioned references for a more thorough description.

\subsection{Numerical scheme and stability}

The steps of the derivation of the DGTD scheme of system (2.3) are well described in [11]. An important point is that the numerical flux, that is introduced through the DGTD scheme, does not need to be modified for the transformed equations. This makes the derivation straightforward.

We consider that $\Omega \subset \mathbb{R}^{3}$ is a bounded convex domain (in fact here, even parallelipipedic as described previously), and $\mathbf{n}$ the unitary outward normal to its boundary $\partial \Omega$. Let $\Omega_{h}$ be a discretization 


\section{Simulating 3D Periodic StruCtures at oblique inCidences}

of $\Omega$, relying on a quasi-uniform triangulation $\mathcal{T}_{h}$ verifying $\overline{\Omega_{h}}=\bigcup_{i=1}^{N} T_{i}$, where $N \in \mathbb{N}^{*}$ is the number of mesh elements, and $\left(T_{i}\right)_{i \in \llbracket 1, N \rrbracket}$ the set of simplices. For each cell $T_{i}, \mathcal{V}_{i}$ is the set of indices $\left\{k \in \llbracket 1, N \rrbracket \mid T_{i} \cap T_{k}\right.$ is a triangular face $\},\left(a_{i l}\right)_{l \in \mathcal{V}_{i}}$ the corresponding faces and $\mathbf{n}_{i l}$ is the unit normal to the face, oriented from $T_{i}$ to $T_{l}$ for $l \in \mathcal{V}_{i}$. Then, let us fix $k>0$ and introduce $\mathcal{V}_{h}$, the subspace of $\mathbf{L}^{2}(\Omega)$ fields that are polynomial of degree at most $k$, on each element of the mesh. For each $W_{h}=\left(W_{h}^{x}, W_{h}^{y}, W_{h}^{z}\right) \in \mathcal{V}_{h}$, we denote by $\mathbf{W}_{i}$, the vector of degrees of freedom on $W_{h}$ restricted to the element $T_{i}$ on the classical Lagrange nodal basis. We suppose that the triangulation is aligned with the various physical media, i.e. an element of the mesh is included in one and only one physical medium.

We use an element-wise weak formulation for (discontinuous) unknowns in $\mathcal{V}_{h}$, where communication within two cells of the mesh is recovered by introducing fluxes. The semi-discrete DG scheme reads in a matricial form: find $\left(\mathbf{P}_{h}, \mathbf{S}_{h}\right) \in \mathcal{V}_{h} \times \mathcal{V}_{h}$ such that for all $i \in\{1, \ldots, N\}$,

$$
\frac{\partial}{\partial t}\left[\begin{array}{c}
\mathbf{P}_{i} \\
\mathbf{S}_{i}
\end{array}\right]=\mathbb{Q}^{-1} \mathbb{M}_{i}^{-1}\left[\begin{array}{r}
-\mathbb{K}_{i} \mathbf{S}_{i}+\sum_{l \in \mathcal{V}_{i}} \mathbb{S}_{i l} \mathbf{S}_{*} \\
\mathbb{K}_{i} \mathbf{P}_{i}-\sum_{l \in \mathcal{V}_{i}} \mathbb{S}_{i l} \mathbf{P}_{*}
\end{array}\right]
$$

where, if we denote for $i \in\{1, \ldots, N\},\left(\phi_{i l}\right)_{l \in\{1, \ldots, d\}}$ the local Lagrange vectorial basis function on the element $T_{i}$ :

- $\mathbb{M}_{i}$ is the local mass matrix on the element $T_{i}$ (of size $\left.d \times d\right)$ with $\left(\mathbb{M}_{i}\right)_{j l}:=\int_{T_{i}} \varepsilon_{r} \phi_{i j} \cdot \phi_{i l}$, $(j, l) \in\{1, \ldots, d\}^{2}$,

- $\mathbb{K}_{i}$ the rigidity matrix on the element $T_{i}($ of size $d \times d)$, with $\left(\mathbb{K}_{i}\right)_{j l}=\int_{T_{i}} \phi_{i j} \cdot \nabla \times \phi_{i l}$, $(j, l) \in\{1, \ldots, d\}^{2}$,

- $\mathbb{S}_{i l}$ the interface matrix, with $\left(\mathbb{S}_{i q}\right)_{j l}=\int_{a_{i q}} \phi_{i j} \cdot \phi_{i l} \times \mathbf{n}_{i q} d s,(j, l) \in\{1, \ldots, d\}^{2}, q \in \mathcal{V}_{i}$

Furthermore, $\mathbf{P}_{*}$ and $\mathbf{S}_{*}$ represent the numerical fluxes on the interfaces [18]:

$$
\mathbf{P}_{*}=\frac{1}{Y_{i}+Y_{l}}\left(\{Y \mathbf{P}\}_{i l}+\alpha \mathbf{n}_{i l} \times \llbracket \mathbf{S} \rrbracket_{i l}\right), \mathbf{S}_{*}=\frac{1}{Z_{i}+Z_{l}}\left(\{Z \mathbf{S}\}_{i l}-\alpha \mathbf{n}_{i l} \times \llbracket \mathbf{P} \rrbracket_{i l}\right) .
$$

on $T_{i} \cap T_{l}, i \in\{1, \ldots, N\}, l \in \mathcal{V}_{i}$. The quantities $Y=\sqrt{\frac{\varepsilon_{r}}{\mu_{r}}}$ and $Z=\frac{1}{Y}$ are respectively the material admittance and impedance. Here $Y_{i}, Z_{i}$ denote their restriction on a triangle $T_{i}$. The parameter $\alpha$ is a given real in $[0,1]$. When $\alpha=0$, one obtains the so-called centered flux. When $\alpha=1$, one obtains the fully upwind flux.

Semi-discrete initial conditions are chosen in accordance with the continuous ones such as $\mathbf{P}_{h}(0, \cdot)=$ $\Pi_{h}(\mathbf{P}(0, \cdot))$ and $\mathbf{S}_{h}(0, \cdot)=\Pi_{h}(\mathbf{S}(0, \cdot))$, with $\Pi_{h}$ a well-chosen projection operator such as the $L^{2}$ one.

Proposition 3.1. Let us suppose that $\left(\boldsymbol{S}_{h}, \boldsymbol{P}_{h}\right) \in \mathcal{C}^{1}\left([0, T], \mathcal{V}_{h}\right) \times \mathcal{C}^{1}\left([0, T], \mathcal{V}_{h}\right)$ is a solution of system (3.1) for a given $\alpha \in[0,1]$. If one defines:

$$
\mathcal{E}_{h}(t):=\frac{1}{2}\left\|\sqrt{\varepsilon_{r}} \boldsymbol{P}_{h}(t, \cdot)\right\|_{\Omega}^{2}+\frac{1}{2}\left\|\sqrt{\mu_{r}} \boldsymbol{S}_{h}(t, \cdot)\right\|_{\Omega}^{2}+\left\langle\frac{\hat{\boldsymbol{k}}_{\|}}{c_{b}} \times \boldsymbol{S}_{h}(t, \cdot), \boldsymbol{P}_{h}(t, \cdot)\right\rangle,
$$

then $\mathcal{E}_{h}$ is a positive quadratic form under the assumption that $\left\|\hat{\boldsymbol{k}}_{\|}\right\|<\min (\bar{c}, 1)$. Furthermore,

$$
\mathcal{E}_{h}(t) \leq \mathcal{E}_{h}(0)+\int_{0}^{t}\left\langle\boldsymbol{S}_{h} \times \boldsymbol{n}, \boldsymbol{P}_{h}\right\rangle_{\Gamma_{e x t}} d s
$$




\section{J. Viquerat, N. Schmitt, et al.}

Proof. We rewrite (3.1) in its variational form. Then the proof is a direct combination of the arguments that appeared in Propositions 2.1 and 2.2 and classical arguments, depending on the fluxes used (see e.g. [8]). We choose not to reproduce it here and will rather focus on the added difficulty of the time discretization.

We directly conclude to the following

Corollary 3.2. If boundary conditions are chosen such that there exists $M>0$ (independent of the discretization parameters) such that $\forall t \in[0, T], \int_{0}^{t}\left\langle\boldsymbol{S}_{h} \times \boldsymbol{n}, \boldsymbol{P}_{h}\right\rangle_{\Gamma_{e x t}} \leq M$, then the semi-discrete scheme is stable.

Remark 3.3. As for the continuous case, the semi-discrete scheme is thus in particular stable if one chooses absorbing boundary conditions or perfect electric conducting boundary conditions.

Regarding the time integration of (3.1), we first propose to investigate the stability of a simple classical explicit scheme (leap-frog with centered fluxes with perfect electric conducting boundary conditions on $\Gamma_{e x t}$, i.e. $P \times \mathbf{n}=0$ on $\left.\Gamma_{e x t}\right)$. in order to understand the impact of the field transform technique on the CFL condition. In the numerical simulations, for accuracy reasons, we would preferably use the well-known five-stages fourth-order Low-Storage Runge-Kutta (LSRK) method described in [2] combined with upwind fluxes $(\alpha=1)$.

We consider a given uniform subdivision of the time interval $[0, T]$ of $J+1$ points $\left(J \in \mathbb{N}^{*}\right)$ and $\Delta t>0$ the corresponding time-step. A leap-frog time integration of (3.1) with centered fluxes (i.e. $\alpha=0$ in the expression of the fluxes (3.2)) gives the fully discrete problem: For all $n \in\{0, \ldots, J\}$, find $\left(\mathbf{P}^{n}, \mathbf{S}^{n+\frac{1}{2}}\right) \in \mathcal{V}_{h} \times \mathcal{V}_{h}$ such that on each element $T_{i}(i \in\{1, \ldots, N\})$ :

$$
\left[\begin{array}{c}
\varepsilon_{r} \frac{\mathbf{P}_{i}^{n+1}-\mathbf{P}_{i}^{n}}{\Delta t}+\frac{\hat{\mathbf{k}}_{\|}}{c_{b}} \times \frac{\mathbf{s}_{i}^{n+\frac{3}{2}}-\mathbf{S}_{i}^{n+\frac{1}{2}}}{\Delta t} \\
\mu_{r} \frac{\mathbf{s}_{i}^{n+\frac{3}{2}}-\mathbf{S}_{i}^{n+\frac{1}{2}}}{\Delta t}-\frac{\hat{\mathbf{k}}_{\|}}{c_{b}} \times \frac{\mathbf{P}_{i}^{n+1}-\mathbf{P}_{i}^{n}}{\Delta t}
\end{array}\right]=\left[\begin{array}{c}
-\mathbb{K}_{i} \mathbf{S}_{i}^{n+\frac{1}{2}}+\sum_{l \in \mathcal{V}_{i}} \mathbb{S}_{i l} \mathbf{S}_{*}^{n+\frac{1}{2}} \\
\mathbb{K}_{i} \mathbf{P}_{i}^{n+1}-\sum_{l \in \mathcal{V}_{i}} \mathbb{S}_{i l} \mathbf{P}_{*}^{n+1}
\end{array}\right],
$$

We define the modified total energy at time-step $n$ as follows:

$$
\mathcal{E}_{h}^{n}:=\frac{1}{2}\left\|\sqrt{\varepsilon_{r}} \mathbf{P}^{n}\right\|_{\Omega}^{2}+\frac{1}{2}\left\langle\mu_{r} \mathbf{S}^{n+\frac{1}{2}}, \mathbf{S}^{n-\frac{1}{2}}\right\rangle_{\Omega}+\frac{1}{2}\left\langle\frac{\hat{\mathbf{k}}_{\|}}{c_{b}} \times \mathbf{S}^{n+\frac{1}{2}}, \mathbf{P}^{n}+\mathbf{P}^{n-1}\right\rangle_{\Omega} .
$$

Proposition 3.4. Assume that $\min (\bar{c}, 1)-\|\hat{\boldsymbol{k}}\|>0$. The energy $\mathcal{E}_{h}^{n}$ is positive under a $C F L$ condition given as

$$
\frac{\Delta t}{h} \leq M \frac{\min (\bar{c}, 1)-\left\|\hat{\boldsymbol{k}}_{\|}\right\|}{c_{b}}
$$

with $M$ a positive generic constant independent of the discretization parameters.

Proof. First we decompose the total energy in a sum of local energies on each element of the mesh. Thus, we denote

$$
\mathcal{E}_{i}^{n}:=\frac{1}{2}\left\|\sqrt{\varepsilon_{r}} \mathbf{P}^{n}\right\|_{T_{i}}^{2}+\frac{1}{2}\left\langle\mu_{r} \mathbf{S}^{n+\frac{1}{2}}, \mathbf{S}^{n-\frac{1}{2}}\right\rangle_{T_{i}}+\frac{1}{2}\left\langle\frac{\hat{\mathbf{k}}_{\|}}{c_{b}} \times \mathbf{S}^{n+\frac{1}{2}}, \mathbf{P}^{n}+\mathbf{P}^{n-1}\right\rangle_{T_{i}},
$$

and we have $\mathcal{E}_{h}^{n}=\sum_{i} \mathcal{E}_{i}^{n}$. And

$$
\begin{aligned}
\mathcal{E}_{i}^{n}=\frac{1}{2}\left\|\sqrt{\varepsilon_{r}} \mathbf{P}^{n}\right\|_{T_{i}}^{2}+\frac{1}{2}\left\|\sqrt{\mu_{r}} \mathbf{S}^{n+\frac{1}{2}}\right\|_{T_{i}}^{2}-\frac{1}{2}\left\langle\mu_{r} \mathbf{S}^{n+\frac{1}{2}}, \mathbf{S}^{n+\frac{1}{2}}-\mathbf{S}^{n-\frac{1}{2}}\right\rangle_{T_{i}} & \\
& +\frac{1}{2}\left\langle\frac{\hat{\mathbf{k}}_{\|}}{c_{b}} \times \mathbf{S}^{n+\frac{1}{2}}, \mathbf{P}^{n}+\mathbf{P}^{n-1}\right\rangle_{T_{i}}
\end{aligned}
$$


Using (3.5), we find

$$
\begin{aligned}
& \frac{1}{2}\left\langle\mu_{r} \mathbf{S}^{n+\frac{1}{2}}, \mathbf{S}^{n+\frac{1}{2}}-\mathbf{S}^{n-\frac{1}{2}}\right\rangle_{T_{i}} \\
& \quad=\frac{1}{2}\left\langle\frac{\hat{\mathbf{k}}_{\|}}{c_{b}} \times\left(\mathbf{P}^{n}-\mathbf{P}^{n-1}\right), \mathbf{S}^{n+\frac{1}{2}}\right\rangle_{T_{i}}+\frac{1}{2} \Delta t\left\langle\mathbf{P}^{n}, \nabla \times \mathbf{S}^{n+\frac{1}{2}}\right\rangle_{T_{i}}-\frac{1}{2} \Delta t\left\langle\mathbf{P}_{*}^{n} \times \mathbf{n}, \mathbf{S}^{n+\frac{1}{2}}\right\rangle_{\partial T_{i}}
\end{aligned}
$$

Combining (3.7) and (3.8), we find

$$
\begin{aligned}
\mathcal{E}_{i}^{n}=\frac{1}{2}\left\|\sqrt{\varepsilon_{r}} \mathbf{P}^{n}\right\|_{T_{i}}^{2}+\frac{1}{2}\left\|\sqrt{\mu_{r}} \mathbf{S}^{n+\frac{1}{2}}\right\|_{T_{i}}^{2}-\frac{1}{2}\left\langle\frac{\hat{\mathbf{k}}_{\|}}{c_{b}} \times\left(\mathbf{P}^{n}-\mathbf{P}^{n-1}\right), \mathbf{S}^{n+\frac{1}{2}}\right\rangle_{T_{i}}-\frac{1}{2} \Delta t\left\langle\mathbf{P}^{n}, \nabla \times \mathbf{S}^{n+\frac{1}{2}}\right\rangle_{T_{i}} \\
+\frac{1}{2} \Delta t\left\langle\mathbf{P}_{*}^{n} \times \mathbf{n}, \mathbf{S}^{n+\frac{1}{2}}\right\rangle_{\partial T_{i}}+\frac{1}{2}\left\langle\frac{\hat{\mathbf{k}}_{\|}}{c_{b}} \times \mathbf{S}^{n+\frac{1}{2}}, \mathbf{P}^{n}+\mathbf{P}^{n-1}\right\rangle_{T_{i}}
\end{aligned}
$$

Thus using boundary conditions,

$$
\begin{aligned}
\mathcal{E}_{i}^{n}=\frac{1}{2}\left\|\sqrt{\varepsilon_{r}} \mathbf{P}^{n}\right\|_{T_{i}}^{2}+\frac{1}{2}\left\|\sqrt{\mu_{r}} \mathbf{S}^{n+\frac{1}{2}}\right\|_{T_{i}}^{2}+\left\langle\frac{\hat{\mathbf{k}}_{\|}}{c_{b}} \times \mathbf{S}^{n+\frac{1}{2}}, \mathbf{P}^{n}\right\rangle_{T_{i}}-\frac{1}{2} \Delta t\left\langle\mathbf{P}^{n}, \nabla \times \mathbf{S}^{n+\frac{1}{2}}\right\rangle_{T_{i}} \\
+\frac{1}{2} \Delta t \sum_{l \in \mathcal{V}_{i}}\left\langle\mathbf{P}_{*}^{n} \times \mathbf{n}, \mathbf{S}^{n+\frac{1}{2}}\right\rangle_{a_{i l}}
\end{aligned}
$$

In a similar manner as in the proof of Proposition 2.8, we have

$$
\begin{aligned}
\sum_{i}\left(\frac{1}{2}\left\|\sqrt{\varepsilon_{r}} \mathbf{P}^{n}\right\|_{T_{i}}^{2}\right. & \left.+\frac{1}{2}\left\|\sqrt{\mu_{r}} \mathbf{S}^{n+\frac{1}{2}}\right\|_{T_{i}}^{2}+\left\langle\frac{\hat{\mathbf{k}}_{\|}}{c_{b}} \times \mathbf{S}^{n+\frac{1}{2}}, \mathbf{P}^{n}\right\rangle_{T_{i}}\right) \\
\geq & \frac{1}{2}\left(1-\frac{\left\|\hat{\mathbf{k}}_{\|}\right\|}{\bar{c}}\right)\left\|\sqrt{\varepsilon_{r}} \mathbf{P}^{n}\right\|_{\Omega_{s}}^{2}+\frac{1}{2}\left(1-\frac{\left\|\hat{\mathbf{k}}_{\|}\right\|}{\bar{c}}\right)\left\|\sqrt{\mu_{r}} \mathbf{S}^{n+\frac{1}{2}}\right\|_{\Omega_{s}}^{2} \\
& +\frac{1}{2}\left(1-\left\|\hat{\mathbf{k}}_{\|}\right\|\right)\left\|\sqrt{\varepsilon_{r}} \mathbf{P}^{n}\right\|_{\Omega_{b}}^{2}+\frac{1}{2}\left(1-\left\|\hat{\mathbf{k}}_{\|}\right\|\right)\left\|\sqrt{\mu_{r}} \mathbf{S}^{n+\frac{1}{2}}\right\|_{\Omega_{b}}^{2} .
\end{aligned}
$$

For the last two terms of (3.10), one makes use of Young's inequality and inverse inequalities stating that there exists $C>0$ independent of the discretization parameters such that for all $\mathbf{U}_{h} \in \mathcal{V}_{h}$,

and

$$
\left\|\nabla \times \mathbf{U}_{h}\right\|_{T_{i}} \leq \frac{C}{h_{i}}\left\|\mathbf{U}_{h}\right\|_{T_{i}}
$$

$$
\left\|\mathbf{U}_{h}\right\|_{\partial T_{i}} \leq \frac{C}{\sqrt{h_{i}}}\left\|\mathbf{U}_{h}\right\|_{T_{i}}
$$

(here $h_{i}$ denotes the diameter of $T_{i}$ ). Using that either $T_{i} \subset \overline{\Omega_{s}}$ or $T_{i} \subset \overline{\Omega_{b}}$, that $\varepsilon_{s}$ is constant on $\Omega_{s}$ and $\Omega_{b}$ and that $\mu_{r} \equiv 1$, we have that there exists $C>0$ such that

$$
\begin{aligned}
-\frac{1}{2} \Delta t\left\langle\mathbf{P}^{n}, \nabla\right. & \left.\times \mathbf{S}^{n+\frac{1}{2}}\right\rangle_{T_{i}}+\frac{1}{2} \Delta t \sum_{l \in \mathcal{V}_{i}}\left\langle\mathbf{P}_{*}^{n} \times \mathbf{n}, \mathbf{S}^{n+\frac{1}{2}}\right\rangle_{a_{i l}} \\
\geq- & \frac{1}{4} C \frac{c_{i, r} \Delta t}{h_{i}}\left\|\sqrt{\varepsilon_{r}} \mathbf{P}^{n}\right\|_{T_{i}}^{2}-\frac{1}{4} C \frac{c_{i, r} \Delta t}{h_{i}}\left\|\sqrt{\mu_{r}} \mathbf{S}^{n+\frac{1}{2}}\right\|_{T_{i}}^{2}-\sum_{l \in \mathcal{V}_{i}}\left(\frac{1}{8} C \frac{c_{i, r} \Delta t}{h_{i}}\left\|\sqrt{\varepsilon_{r}} \mathbf{P}^{n}\right\|_{T_{i}}^{2}\right. \\
& \left.+\frac{1}{8} C \frac{c_{i, r} \Delta t}{h_{i}}\left\|\sqrt{\mu_{r}} \mathbf{S}^{n+\frac{1}{2}}\right\|_{T_{i}}^{2}+\frac{1}{8} C \frac{c_{l, r} \Delta t}{h_{i}}\left\|\sqrt{\varepsilon_{r}} \mathbf{P}^{n}\right\|_{T_{l}}^{2}+\frac{1}{8} C \frac{c_{l, r} \Delta t}{h_{i}}\left\|\sqrt{\mu_{r}} \mathbf{S}^{n+\frac{1}{2}}\right\|_{T_{l}}^{2}\right),
\end{aligned}
$$

with $c_{k, r}=c_{s}$ or $c_{k, r}=c_{b}$ with $k \in\{l, i\}$ depending on the physical domain where $T_{k}$ is included. 


\section{J. Viquerat, N. Schmitt, et al.}

Finally, summing over all mesh elements and using quasi-uniformity of the mesh, one has that there exists $M>0$ such that

$$
\begin{aligned}
& \sum_{i}\left(-\frac{1}{2} \Delta t\left\langle\mathbf{P}^{n}, \nabla \times \mathbf{S}^{n+\frac{1}{2}}\right\rangle_{T_{i}}\right.\left.+\frac{1}{2} \Delta t \sum_{l \in \mathcal{V}_{i}}\left\langle\mathbf{P}_{*}^{n} \times \mathbf{n}, \mathbf{S}^{n+\frac{1}{2}}\right\rangle_{a_{i l}}\right) \\
& \geq-\frac{1}{2} M \frac{c_{b} \Delta t}{h}\left\|\sqrt{\varepsilon_{b}} \mathbf{P}^{n}\right\|_{\Omega_{b}}^{2}-\frac{1}{2} M \frac{c_{b} \Delta t}{h}\left\|\sqrt{\mu_{b}} \mathbf{S}^{n+\frac{1}{2}}\right\|_{\Omega_{b}}^{2} \\
& \quad-\frac{1}{2} M \frac{c_{s} \Delta t}{h}\left\|\sqrt{\varepsilon_{s}} \mathbf{P}^{n}\right\|_{\Omega_{s}}^{2}-\frac{1}{2} M \frac{c_{s} \Delta t}{h}\left\|\sqrt{\mu_{s}} \mathbf{S}^{n+\frac{1}{2}}\right\|_{\Omega_{s}}^{2} .
\end{aligned}
$$

Combining this last estimate with (3.11) leads to

$$
\begin{aligned}
\mathcal{E}^{n} \geq \frac{1}{2}(1-M & \left.\frac{c_{b} \Delta t}{h}-\left\|\hat{\mathbf{k}}_{\|}\right\|\right)\left\|\sqrt{\varepsilon_{b}} \mathbf{P}^{n}\right\|_{\Omega_{b}}^{2}+\frac{1}{2}\left(1-M \frac{c_{b} \Delta t}{h}-\left\|\hat{\mathbf{k}}_{\|}\right\|\right)\left\|\sqrt{\mu_{b}} \mathbf{S}^{n+\frac{1}{2}}\right\|_{\Omega_{b}}^{2} \\
+ & \frac{1}{2}\left(1-M \frac{c_{s} \Delta t}{h}-\frac{\left\|\hat{\mathbf{k}}_{\|}\right\|}{\bar{c}}\right)\left\|\sqrt{\varepsilon_{s}} \mathbf{P}^{n}\right\|_{\Omega_{s}}^{2}+\frac{1}{2}\left(1-M \frac{c_{s} \Delta t}{h}-\frac{\left\|\hat{\mathbf{k}}_{\|}\right\|}{\bar{c}}\right)\left\|\sqrt{\mu_{s}} \mathbf{S}^{n+\frac{1}{2}}\right\|_{\Omega_{s}}^{2} .
\end{aligned}
$$

This implies that if

$$
\frac{\Delta t}{h} \leq M^{-1} \frac{\min (\bar{c}, 1)-\left\|\hat{\mathbf{k}}_{\|}\right\|}{c_{b}}
$$

then the energy is positive.

Proposition 3.5. For all $n \in\{0, \ldots, J-1\}$, the modified energy $\mathcal{E}_{h}^{n+\frac{1}{2}}$ verifies a discrete energy principle,

$$
\mathcal{E}_{h}^{n+1}-\mathcal{E}_{h}^{n}=0 .
$$

Proof. Let $n \in\{0, \ldots, J-1\}$. Using the scheme with the adequate test functions $\mathbf{P}^{n+1}+\mathbf{P}^{n}$ and $\mathbf{S}^{n+\frac{1}{2}}$ in respectively the first and second equation (at time $n$ and $n+1$ for the second equation) of (3.5), one obtains:

$$
\begin{gathered}
\left\|\sqrt{\varepsilon_{r}} \mathbf{P}^{n+1}\right\|_{T_{i}}^{2}-\left\|\sqrt{\varepsilon_{r}} \mathbf{P}^{n}\right\|_{T_{i}}^{2}+\left\langle\frac{\hat{\mathbf{k}}_{\|}}{c_{b}} \times\left(\mathbf{S}^{n+\frac{3}{2}}-\mathbf{S}^{n+\frac{1}{2}}\right), \mathbf{P}^{n+1}+\mathbf{P}^{n}\right\rangle_{T_{i}} \\
=\Delta t\left\langle-\mathbf{S}^{n+\frac{1}{2}}, \nabla \times\left(\mathbf{P}^{n+1}+\mathbf{P}^{n}\right)\right\rangle_{T_{i}}+\Delta t \sum_{l \in \mathcal{V}_{i}}\left\langle\mathbf{S}_{*}^{n+\frac{1}{2}} \times \mathbf{n}, \mathbf{P}^{n+1}+\mathbf{P}^{n}\right\rangle_{a_{i l}}, \\
\left\langle\mu_{r}\left(\mathbf{S}^{n+\frac{1}{2}}-\mathbf{S}^{n-\frac{1}{2}}\right), \mathbf{S}^{n+\frac{1}{2}}\right\rangle_{T_{i}}-\left\langle\frac{\hat{\mathbf{k}}_{\|}}{c_{b}} \times\left(\mathbf{P}^{n}-\mathbf{P}^{n-1}\right), \mathbf{S}^{n+\frac{1}{2}}\right\rangle_{T_{i}} \\
=\Delta t\left\langle\mathbf{P}^{n}, \nabla \times \mathbf{S}^{n+\frac{1}{2}}\right\rangle_{T_{i}}-\Delta t \sum_{l \in \mathcal{V}_{i}}\left\langle\mathbf{P}_{*}^{n} \times \mathbf{n}, \mathbf{S}^{n+\frac{1}{2}}\right\rangle_{a_{i l}} \\
\left\langle\mu_{r}\left(\mathbf{S}^{n+\frac{3}{2}}-\mathbf{S}^{n+\frac{1}{2}}\right), \mathbf{S}^{n+\frac{1}{2}}\right\rangle_{T_{i}}-\left\langle\frac{\hat{\mathbf{k}}_{\|}}{c_{b}} \times\left(\mathbf{P}^{n+1}-\mathbf{P}^{n}\right), \mathbf{S}^{n+\frac{1}{2}}\right\rangle_{T_{i}} \\
=\Delta t\left\langle\mathbf{P}^{n+1}, \nabla \times \mathbf{S}^{n+\frac{1}{2}}\right\rangle_{T_{i}}-\Delta t \sum_{l \in \mathcal{V}_{i}}\left\langle\mathbf{P}_{*}^{n+1} \times \mathbf{n}, \mathbf{S}^{n+\frac{1}{2}}\right\rangle_{a_{i l}} .
\end{gathered}
$$


We add the three resulting equations and rewrite them using the properties of the mixed product:

$$
\begin{aligned}
\left\|\sqrt{\varepsilon_{r}} \mathbf{P}^{n+1}\right\|_{T_{i}}^{2}+\left\langle\frac{\hat{\mathbf{k}}_{\|}}{c_{b}} \times\left(\mathbf{S}^{n+\frac{3}{2}}-\mathbf{S}^{n+\frac{1}{2}}\right), \mathbf{P}^{n+1}+\mathbf{P}^{n}\right\rangle_{T_{i}}+\left\langle\mu_{r} \mathbf{S}^{n+\frac{3}{2}}, \mathbf{S}^{n+\frac{1}{2}}\right\rangle_{T_{i}} \\
-\left\|\sqrt{\varepsilon_{r}} \mathbf{P}^{n}\right\|_{T_{i}}^{2}-\left\langle\frac{\hat{\mathbf{k}}_{\|}}{c_{b}} \times\left(\mathbf{P}^{n+1}-\mathbf{P}^{n-1}\right), \mathbf{S}^{n+\frac{1}{2}}\right\rangle_{T_{i}}-\left\langle\mu_{r} \mathbf{S}^{n+\frac{1}{2}}, \mathbf{S}^{n-\frac{1}{2}}\right\rangle_{T_{i}} \\
=-\Delta t\left\langle\mathbf{S}^{n+\frac{1}{2}}, \nabla \times\left(\mathbf{P}^{n+1}+\mathbf{P}^{n}\right)\right\rangle_{T_{i}}+\Delta t \sum_{l \in \mathcal{V}_{i}}\left\langle\mathbf{S}_{*}^{n+\frac{1}{2}} \times \mathbf{n}, \mathbf{P}^{n+1}+\mathbf{P}^{n}\right\rangle_{a_{i l}} \\
+\Delta t\left\langle\mathbf{P}^{n}+\mathbf{P}^{n+1}, \nabla \times \mathbf{S}^{n+\frac{1}{2}}\right\rangle_{T_{i}}-\Delta t \sum_{l \in \mathcal{V}_{i}}\left\langle\mathbf{P}_{*}^{n}+\mathbf{P}_{*}^{n+1} \times \mathbf{n}, \mathbf{S}^{n+\frac{1}{2}}\right\rangle_{a_{i l}} .
\end{aligned}
$$

Let $I_{i}$ be the right hand side of this equality and using integration by part formula and the expression of the centered flux, we obtain

$$
\sum_{i} I_{i}:=0
$$

Furthermore

$$
\begin{aligned}
\left\langle\frac{\hat{\mathbf{k}}_{\|}}{c_{b}} \times\left(\mathbf{S}^{n+\frac{3}{2}}-\mathbf{S}^{n+\frac{1}{2}}\right), \mathbf{P}^{n+1}+\right. & \left.\mathbf{P}^{n}\right\rangle_{T_{i}}-\left\langle\frac{\hat{\mathbf{k}}_{\|}}{c_{b}} \times\left(\mathbf{P}^{n+1}-\mathbf{P}^{n-1}\right), \mathbf{S}^{n+\frac{1}{2}}\right\rangle_{T_{i}} \\
& =\left\langle\frac{\hat{\mathbf{k}}_{\|}}{c_{b}} \times \mathbf{S}^{n+\frac{3}{2}}, \mathbf{P}^{n+1}+\mathbf{P}^{n}\right\rangle_{T_{i}}\left\langle\frac{\hat{\mathbf{k}}_{\|}}{c_{b}} \times \mathbf{S}^{n+\frac{1}{2}}, \mathbf{P}^{n}+\mathbf{P}^{n-1}\right\rangle_{T_{i}}
\end{aligned}
$$

Summing over all the elements, taking into account all the contributions, we find:

$$
\mathcal{E}_{h}^{n+1}-\mathcal{E}_{h}^{n}=0 .
$$

The last result together with Proposition 3.4, leads to the stability of the scheme under the CFL condition of Proposition 3.4.

The same kind of analysis can be adapted to take into account for the upwind fluxes and absorbing boundary conditions (the latter have only negative contributions in the energy principle), and to more complicated schemes such as explicit Runge-Kutta type time discretization scheme. This could be obtained by adapting the arguments of this paper and those of [8]. However, this would end up in a lengthy, tedious and technical proof that we choose not to reproduce here. In the simulations, we will mostly use explicit LSRK schemes with fully upwind fluxes (as mentioned earlier) and we will verify empirically the accuracy of the above CFL condition.

Remark 3.6. As a side note, we would like to notice that one could be tempted to discretize system (3.1) as follows with a LSRK scheme:

$$
\left[\begin{array}{c}
\partial_{t} \mathbf{P}_{i} \\
\partial_{t} \mathbf{S}_{i}
\end{array}\right]^{n+1}=\mathbb{M}_{i}^{-1}\left[\begin{array}{r}
-\mathbb{K}_{i} \mathbf{S}_{i}+\sum_{l \in \mathcal{V}_{i}} \mathbb{S}_{i l} \mathbf{S}_{*} \\
\mathbb{K}_{i} \mathbf{P}_{i}-\sum_{l \in \mathcal{V}_{i}} \mathbb{S}_{i l} \mathbf{P}_{*}
\end{array}\right]^{n}+\left[\begin{array}{r}
-\hat{\mathbf{k}}_{\|} \times \partial_{t} \mathbf{P}_{i} \\
\hat{\mathbf{k}}_{\|} \times \partial_{t} \mathbf{S}_{i}
\end{array}\right]^{n},
$$

the additional time-derivative term being stored at each time-step and acting a source on the righthand side for the computation of the next time-derivative of the fields. Although this method leads to a stable and valid discretization, the stability constraints with increasing angles are much worse than that obtained with time integration of the scheme (3.1) using LSRK4, leading to unbearably long computation times.

We now specify the type of sources and boundary conditions that we are using. 


\subsection{Incident field}

When implementing the field transform equations into a time-domain solver, care must be taken to adapt the expressions of the source fields to the new formulation. Here, we cover the well-known case of the wide-band plane wave source in a background material. It reads for respectively the electric and magnetic fields

$$
\begin{aligned}
\mathbf{E}_{\mathrm{inc}}(\mathbf{r}, t) & =\mathbf{E}_{0} \sin \left(\omega_{0}\left(t-\frac{\hat{\mathbf{k}} \cdot \mathbf{r}}{c_{b}}\right)\right) \exp \left(-\frac{\left(t-\frac{\hat{\mathbf{k}} \cdot \mathbf{r}}{c_{b}}\right)^{2}}{2 \sigma^{2}}\right), \\
\mathbf{H}_{\mathrm{inc}} & =\frac{\hat{\mathbf{k}} \times \mathbf{E}_{\mathrm{inc}}}{Z_{b}}, \\
\hat{\mathbf{k}} \cdot \mathbf{E}_{0} & =0
\end{aligned}
$$

where $\omega_{0}$ is the central frequency of the pulse, $\sigma$ controls the width of the Gaussian (and hence the spectral range), and $Z_{b}$ is the optical impedance of the background material. The third equation expresses that the polarization and the wavevector must be orthogonal. Applying transformation (2.2) to the frequency-domain version of system (3.19) and transforming back to time-domain is straightforward:

$$
\begin{aligned}
\mathbf{P}_{\text {inc }}(\mathbf{r}, t) & =\mathbf{P}_{0} \sin \left(\omega_{0}\left(t-\frac{\hat{\mathbf{k}}_{\perp} \cdot \mathbf{r}}{c_{b}}\right)\right) \exp \left(-\frac{\left(t-\frac{\hat{\mathbf{k}}_{\perp} \cdot \mathbf{r}}{c_{b}}\right)^{2}}{2 \sigma^{2}}\right), \\
\mathbf{S}_{\mathrm{inc}} & =\frac{\hat{\mathbf{k}} \times \mathbf{P}_{\text {inc }}}{Z_{b}}, \\
\hat{\mathbf{k}} \cdot \mathbf{P}_{0} & =0 .
\end{aligned}
$$

Care must be taken that the transformation does not affect the second and third relations, where the full wavevector still appears.

\subsection{CFS-PML}

We now turn to the treatment of the exterior boundary. As mentioned earlier, we have to artificially truncate the domain in the non-periodic directions. We could have used absorbing boundary conditions, but we rather choose to add a PML layer at the artificial boundary that will absorb the field scattered by the structure. Initially designed by Kuzuoglu et al. [7], the first adaptation of CFS-PMLs to the DGTD framework was recently proposed in [6]. These PMLs rely on a complex stretching of the spatial coordinates:

$$
\frac{\partial}{\partial k} \rightarrow \frac{1}{s_{k}(\omega)} \frac{\partial}{\partial k} \text { with } s_{k}(\omega)=1-\frac{\sigma_{k}}{i \omega-\alpha_{k}} \text { and } k \in\{x, y, z\}
$$

In the latter expression, $\sigma_{k}$ represents the damping rate of the PML, while $\alpha_{k}$ is the actual frequency shift. In the original expression of the CFS-PMLs, a real stretch was also present, but it was removed here for the sake of simplicity. The reader is referred to [6] for additional details about the CFS-PML. On the PML layer, the modified Maxwell's equations for the scattered field under this stretch are given 
by (see $[6])$ :

$$
\begin{aligned}
\varepsilon_{r} \frac{\partial \mathbf{E}}{\partial t} & =\nabla \times \mathbf{H}-\mathbf{G}_{+}^{E}-\mathbf{G}_{-}^{E}, \\
\frac{\partial \mathbf{G}_{+}^{E}}{\partial t} & =\boldsymbol{\sigma}_{+} \odot \nabla_{+} \times \mathbf{H}-\left(\boldsymbol{\alpha}_{+}+\boldsymbol{\sigma}_{+}\right) \odot \mathbf{G}_{+}^{E}, \\
\frac{\partial \mathbf{G}_{-}^{E}}{\partial t} & =-\boldsymbol{\sigma}_{-} \odot \nabla_{-} \times \mathbf{H}-\left(\boldsymbol{\alpha}_{-}+\boldsymbol{\sigma}_{-}\right) \odot \mathbf{G}_{-}^{E}, \\
\mu_{r} \frac{\partial \mathbf{H}}{\partial t} & =-\nabla \times \mathbf{E}-\mathbf{G}_{+}^{H}-\mathbf{G}_{-}^{H}, \\
\frac{\partial \mathbf{G}_{+}^{H}}{\partial t} & =-\boldsymbol{\sigma}_{+} \odot \nabla_{+} \times \mathbf{E}-\left(\boldsymbol{\alpha}_{+}+\boldsymbol{\sigma}_{+}\right) \odot \mathbf{G}_{+}^{H}, \\
\frac{\partial \mathbf{G}_{-}^{H}}{\partial t} & =\boldsymbol{\sigma}_{-} \odot \nabla_{-} \times \mathbf{E}-\left(\boldsymbol{\alpha}_{-}+\boldsymbol{\sigma}_{-}\right) \odot \mathbf{G}_{-}^{H},
\end{aligned}
$$

where the following notations are used:

$$
\nabla_{+} \times \mathbf{U}=\left[\begin{array}{c}
\partial_{y} U_{z} \\
\partial_{z} U_{x} \\
\partial_{x} U_{y}
\end{array}\right], \nabla_{-} \times \mathbf{U}=\left[\begin{array}{c}
\partial_{z} U_{y} \\
\partial_{x} U_{z} \\
\partial_{y} U_{x}
\end{array}\right], \boldsymbol{\sigma}_{+}=\left[\begin{array}{c}
\sigma_{y} \\
\sigma_{z} \\
\sigma_{x}
\end{array}\right], \boldsymbol{\sigma}_{-}=\left[\begin{array}{c}
\sigma_{z} \\
\sigma_{x} \\
\sigma_{y}
\end{array}\right]
$$

and similarly for $\boldsymbol{\alpha}_{+}$and $\boldsymbol{\alpha}_{-}$. The notation $\odot$ designates the element-wise multiplication for vectors, while $\mathbf{G}_{+}^{U}$ and $\mathbf{G}_{-}^{U}$ are additional fields required for the resolution of the field damping within the PML region. Applying the field transformation (2.2) to the full system of Maxwell's equations with CFS-PMLs would lead to a very large, tangled system with 18 equations, thus considerably enlarging the size of matrix $\mathbb{Q}$. To get around this problem, it is however possible to apply the coordinate stretching (3.20) directly to the transformed system (2.3). Since the modifications brought by the stretching only impact the spatial derivatives, the standard derivation can be followed as in [6], and the final continuous system of equations reads (again for the scattered field):

$$
\begin{aligned}
\varepsilon_{r} \frac{\partial \mathbf{P}}{\partial t}+\frac{\hat{\mathbf{k}}_{\|}}{c_{b}} \times \frac{\partial \mathbf{S}}{\partial t} & =\nabla \times \mathbf{S}-\mathbf{G}_{+}^{P}-\mathbf{G}_{-}^{P}, \\
\frac{\partial \mathbf{G}_{+}^{P}}{\partial t} & =\boldsymbol{\sigma}_{+} \odot \nabla_{+} \times \mathbf{S}-\left(\boldsymbol{\alpha}_{+}+\boldsymbol{\sigma}_{+}\right) \odot \mathbf{G}_{+}^{P}, \\
\frac{\partial \mathbf{G}_{-}^{P}}{\partial t} & =-\boldsymbol{\sigma}_{-} \odot \nabla_{-} \times \mathbf{S}-\left(\boldsymbol{\alpha}_{-}+\boldsymbol{\sigma}_{-}\right) \odot \mathbf{G}_{-}^{P}, \\
\mu_{r} \frac{\partial \mathbf{S}}{\partial t}-\frac{\hat{\mathbf{k}}_{\|}}{c_{b}} \times \frac{\partial \mathbf{P}}{\partial t} & =-\nabla \times \mathbf{P}-\mathbf{G}_{+}^{S}-\mathbf{G}_{-}^{S}, \\
\frac{\partial \mathbf{G}_{+}^{S}}{\partial t} & =-\boldsymbol{\sigma}_{+} \odot \nabla_{+} \times \mathbf{P}-\left(\boldsymbol{\alpha}_{+}+\boldsymbol{\sigma}_{+}\right) \odot \mathbf{G}_{+}^{S}, \\
\frac{\partial \mathbf{G}_{-}^{S}}{\partial t} & =\boldsymbol{\sigma}_{-} \odot \nabla_{-} \times \mathbf{P}-\left(\boldsymbol{\alpha}_{-}+\boldsymbol{\sigma}_{-}\right) \odot \mathbf{G}_{-}^{S} .
\end{aligned}
$$

After discretization, the CFS-PML equations can be solved as they are with the standard Maxwell's scheme (see [18]), while the damping fields $\mathbf{G}_{+}^{U}$ and $\mathbf{G}_{-}^{U}$ (for $U \in\{\mathbf{P}, \mathbf{S}\}$ ) only need to be incorporated to the RHS of the $\mathbf{P}$ and $\mathbf{S}$ update equations. The reader is referred to [6] and [18] for the details of the DG discretization of CFS-PMLs. 


\subsection{Total Field/Scattered Field (TF/SF) formulation.}

The combination of the absorbing PML layer and the incident field is dealt with by using a classical TF/SF strategy that exploits the possibility of splitting the contribution of the fields in their incident and scattered parts. In this manner, the computational domain is split into two parts: one containing the main scatterer where the total field is computed and the other complementary part where only the scattered field is computed (thus including the CFS PML zone). The incident field is in consequence imposed through the TF/SF interface that delimits these two zones (see e.g. [18] for more details) and figure 1.1 for the whole picture.

\subsection{Incorporation of dispersion models}

At the frequency range considered in nanophotonics, the impact of dispersion can not be neglected in metallic media. The electrons don't instantaneously react to the applied electric field. This is rendered via frequency dependent permittivity laws, and thus frequency dependent speed of propagation of a given incident wave. In the temporal domain, this behavior is expressed via the introduction of one (or possibly multiple) polarization current(s). In the simplest version of a dispersion model (that is the framework used in this work), the evolution of the polarization currents is driven by auxiliary ODE's that are linearly coupled to Maxwell's equation as source currents.

For the sake of clarity, we recall the most classical version of such dispersion models called the generalized dispersion model.

$$
\begin{array}{rlrl}
\mu_{0} \partial_{t} \mathbf{H} & =-\nabla \times \mathbf{E}, & \\
\varepsilon_{0} \varepsilon_{s} \partial_{t} \mathbf{E} & =\nabla \times \mathbf{H}-\mathbf{J}_{\mathrm{f}}-\mathbf{J}_{\mathrm{b}}, & \\
\partial_{t} \mathbf{J}_{\mathrm{f}} & =-\gamma \mathbf{J}_{\mathrm{f}}+\omega_{\mathrm{P}}^{2} \varepsilon_{0} \mathbf{E}, & \\
\mathbf{J}_{\mathrm{b}} & =\mathcal{J}_{0}+\sum_{i \in L_{1}} \mathcal{J}_{i}+\sum_{i \in L_{2}} \mathcal{J}_{i}, & & \\
\mathcal{J}_{0} & =\left(\sigma+\sum_{i \in L_{2}} \mathcal{J}_{i}\right) \mathbf{E}, & \\
\mathcal{J}_{i} & =a_{i} \mathbf{E}-b_{i} \mathcal{P}_{i}, & & \forall i \in L_{1}, \\
\partial_{t} \mathcal{P}_{i} & =\mathcal{J}_{i}, & & \forall i \in L_{1}, \\
\partial_{t} \mathcal{J}_{i} & =\left(c_{i}-d_{i} f_{i}\right) \mathbf{E}-f_{i} \mathcal{J}_{i}-e_{i} \mathcal{P}_{i}, & \forall i \in L_{2}, \\
\partial_{t} \mathcal{P}_{i} & =d_{i} \mathbf{E}+\mathcal{J}_{i}, & & \forall i \in L_{2} .
\end{array}
$$

The fields $\mathbf{J}_{\mathrm{f}}$ and $\mathbf{J}_{\mathrm{b}}$ are the polarization currents of respectively the free and bound electrons. The parameters $\gamma, \omega_{\mathrm{P}}^{2}$ and $\sigma$ are given physical parameters. The bound polarization decouples into several polarization currents. Their evolution is given using the set of parameters $\left(a_{i}, b_{i}\right)_{i \in L_{1}},\left(c_{i}, d_{i}, e_{i}, f_{i}\right)_{i \in L_{2}}$, that are given parameters, used to design the model according to experimental data. The classical Drude model, as referred to in this paper, corresponds to the case where $\mathbf{J}_{b}=0$.

We refer the reader to $[18,8]$ for more details.

In the sequel for realistic numerical simulations, we will rely on such models. The extension of the field-transform technique is straightforward in this case. 


\section{Numerical results}

We now concentrate on the numerical results. We will first provide some useful observables. Then, we illustrate the behavior inferred from the theoretical results and then tackle several nanophotonic test cases.

\subsection{Observables}

A few observables are usually at the heart of the analysis of periodic structures. In this section, we shortly review some of them, and see how they transform in the case of oblique incidence.

\subsubsection{Reflection and tranmission}

The most common quantities of interest for periodic structures are certainly the reflectance $R$ and the transmittance $T$, which are frequency-dependent quantities that provide informations about the capability of a structure to reflect or let power flow through it. They are usually computed from the time-averaged Poynting vector:

$$
\boldsymbol{\pi}=\frac{1}{2} \Re\left(\hat{\mathbf{E}} \times \hat{\mathbf{H}}^{*}\right)
$$

where we denote by $\hat{\cdot}$ the Fourier transform.

Integrated over proper monitoring surfaces, this leads to the knowledge of $R$ and $T$ :

$$
R(\omega)=\frac{\int_{S_{i}} \boldsymbol{\pi}_{\mathrm{sca}} \cdot \mathbf{n}}{\int_{S_{i}} \boldsymbol{\pi}_{\mathrm{inc}} \cdot \mathbf{n}}, T(\omega)=\frac{\int_{S_{o}} \boldsymbol{\pi}_{\mathrm{tot}} \cdot \mathbf{n}}{\int_{S_{i}} \boldsymbol{\pi}_{\mathrm{inc}} \cdot \mathbf{n}},
$$

where the subscripts refer to scattered, incident and total field.

Thanks to the conjugate in expression (4.1), applying the field transform (2.2) simply leads to:

$$
\boldsymbol{\pi}=\frac{1}{2} \Re\left(\hat{\mathbf{P}} \times \hat{\mathbf{S}}^{*}\right) .
$$

\subsubsection{Diffraction efficiency}

A particularly important class of periodic structures is that of gratings, which have the ability to diffract incident light in a discrete set of beams, each one propagating in a specific direction. The functioning of a grating is usually characterized by the computation of its diffraction efficiencies $\eta_{n, m}$, which describe how the transmitted (or reflected) power divides between the existing diffraction orders [12]. Assuming a doubly periodic domain with lateral sizes $a_{x}$ and $a_{y}$, the propagative scattered far field at a fixed height $z_{0}$ above the grating can be decomposed on a Fourier basis:

$$
\begin{aligned}
\hat{\mathbf{E}}_{\mathrm{sca}}(\mathbf{r}, \omega) & =\sum_{(n, m) \in \mathbb{Z}^{2}} \mathbf{e}_{n, m}(\omega) \exp \left[i\left(\mathbf{k}_{x}^{\mathrm{sca}} x+\mathbf{k}_{y}^{\mathrm{sca}} y\right)\right] \\
& =\sum_{(n, m) \in \mathbb{Z}^{2}} \mathbf{e}_{n, m}(\omega) \exp \left[i\left(\left(\mathbf{k}_{x}+\mathbf{k}_{n}\right) x+\left(\mathbf{k}_{y}+\mathbf{k}_{m}\right) y\right)\right],
\end{aligned}
$$

where we defined $\mathbf{k}_{n}=\frac{2 \pi n}{a_{x}}, \mathbf{k}_{m}=\frac{2 \pi m}{a_{y}}$. In expression (4.3), each couple $(n, m)$ contributes to the sum under the condition that:

$$
\mathbf{k}_{z}^{\mathrm{sca}}=\sqrt{|\mathbf{k}|^{2}-\left(\mathbf{k}_{x}+\mathbf{k}_{n}\right)^{2}-\left(\mathbf{k}_{y}+\mathbf{k}_{m}\right)^{2}} \in \mathbb{R},
$$


meaning that only propagative modes can contribute to the far field. The complex Fourier coefficients are defined by:

$$
\mathbf{e}_{n, m}(\omega)=\left.\frac{1}{a_{x} a_{y}} \int_{a_{x}} \int_{a_{y}} \hat{\mathbf{E}}(\mathbf{r}, \omega)\right|_{z_{0}} \exp \left[-i\left(\left(\mathbf{k}_{x}+\mathbf{k}_{n}\right) x+\left(\mathbf{k}_{y}+\mathbf{k}_{m}\right) y\right)\right] \mathrm{d} x \mathrm{~d} y .
$$

Analogous steps apply for the $\mathbf{H}$ field, leading to a similar expression for $\mathbf{h}_{n, m}(\omega)$. For each propagative mode, an associated Poynting vector can be computed:

$$
\boldsymbol{\pi}_{n, m}(\omega)=\frac{1}{2} \Re\left(\mathbf{e}_{n, m}(\omega) \times \mathbf{h}_{n, m}^{*}(\omega)\right),
$$

from which the mode-wise diffraction efficiency can be defined:

$$
\eta_{n, m}(\omega)=a_{x} a_{y} \boldsymbol{\pi}_{n, m}(\omega) .
$$

The diffraction efficiency is related to the reflectance as follows, under condition (4.4):

$$
R(\omega)=\sum_{(n, m) \in \mathbb{Z}^{2}} \eta_{n, m}(\omega),
$$

As can be seen from expressions (4.6) and (4.7), the definition of $\eta_{n, m}$ remains unchanged under transformation (2.2):

$$
\boldsymbol{\pi}_{n, m}(\omega)=\frac{1}{2} \Re\left(\mathbf{p}_{n, m}(\omega) \times \mathbf{s}_{n, m}^{*}(\omega)\right),
$$

where $\mathbf{p}_{n, m}$ and $\mathbf{s}_{n, m}$ are respectively defined using expression (4.5) replacing $\hat{E}$ with respectively $\hat{P}$ and $\hat{S}$.

Remark 4.1. All these quantities are here defined as functions of frequency. However, in the numerical results sections, these latter will be instead represented as functions of wavelength. Abusing the notations, we will still write $R(\lambda), \eta_{n, m}(\lambda)$ etc.

\subsection{Time-step reduction}

As a first validation of the results of section 2.2, we consider a bi-periodic domain of lateral size 75 $\mathrm{nm}$ that consists of vacuum, terminated with CFS-PMLs regions at the top and bottom. A broadband plane wave with significant intensity in the range $\lambda \in[150,600] \mathrm{nm}$ is injected through a TotalField/Scattered-Field (TF/SF) interface, and the quantity $\overline{\Delta t}=\frac{\Delta t\left(\theta=0^{\circ}\right)}{\Delta t(\theta)}$ for $\theta \in\left[0^{\circ}, 90^{\circ}\right.$ is computed, where $\Delta t(\theta)$ denotes the maximal stable time-step found for a given angle $\theta$. The scheme used is LSRK4 with upwind fluxes $(\alpha=1)$. The results, obtained for piecewise $\mathbb{P}_{3}$ polynomial approximation, are shown in figure 4.1 along with $\lambda_{x^{+}}$. Although there is not a perfect match, the general trend confirms our previous analysis. The deviation observed for large incidence angles can be attributed to the dissipation induced by the fully upwind scheme that stabilizes the numerical algorithm.

\subsection{Critical angle with dielectric background}

We now consider a slab of vacuum $\left(c_{r}=1\right)$ embedded in a dielectric background for which $c_{b}=\frac{1}{\sqrt{2}}$, i.e. $\varepsilon_{b}=2$. In this case, the expected critical angle is $\theta_{c}=45^{\circ}$. The rest of the configuration is similar to that of last section. In figure 4.2, we plot the figure of merit $\overline{\Delta t}$ : as before, although the match is not perfect, the trend of the numerical results decently follows the theory. As it was suggested in section 2.2, no stable time-step can be found for $\theta>\theta_{c}$, confirming the previous analysis. 


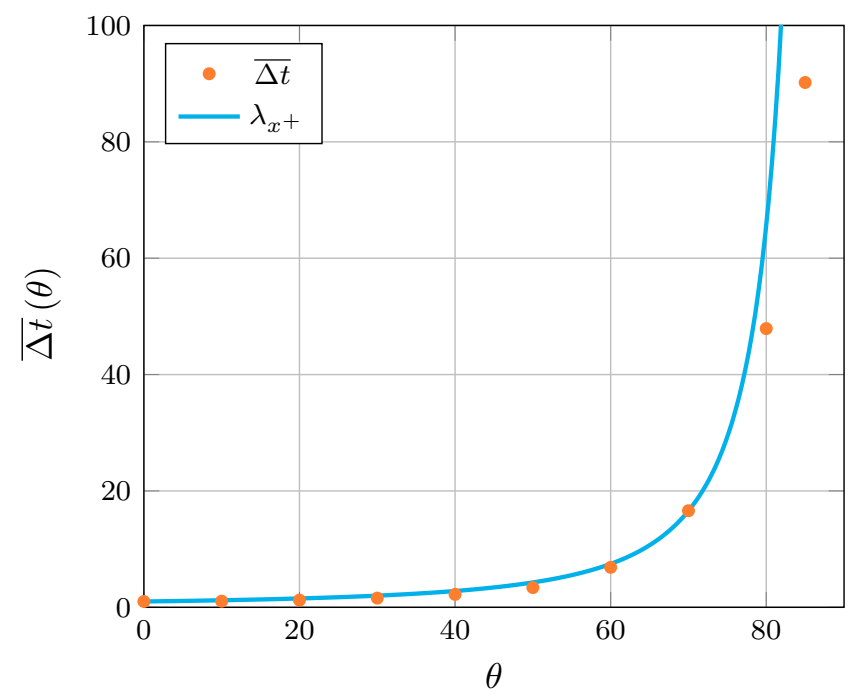

FIgURE 4.1. Relative maximal stable timestep $\overline{\Delta t}=\frac{\Delta t\left(\theta=0^{\circ}\right)}{\Delta t(\theta)}$ for $\theta \in\left[0^{\circ}, 90^{\circ}[\right.$ compared to $\lambda_{x^{+}}$in vacuum.

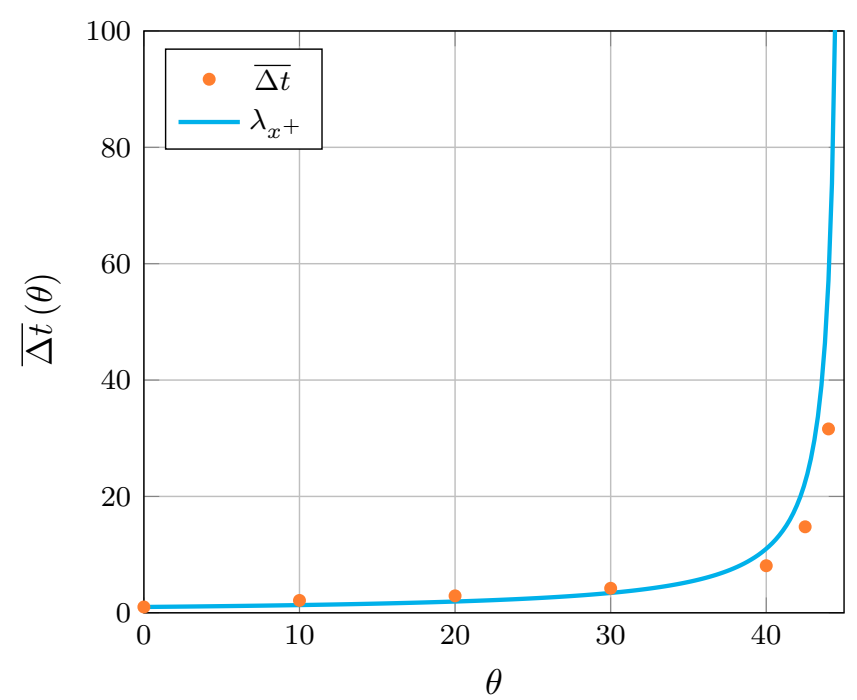

FIGURE 4.2. Relative maximal stable timestep $\overline{\Delta t}=\frac{\Delta t\left(\theta=0^{\circ}\right)}{\Delta t(\theta)}$ for $\theta \in\left[0^{\circ}, 45^{\circ}[\right.$ compared to $\lambda_{x^{+}}$for a slab of vacuum embedded between two dielectric half-spaces.

\subsection{Bi-periodic gold slab}

We consider the case of a bi-periodic gold slab of thickness $50 \mathrm{~nm}$ embedded in vacuum and illuminated by a wide-band plane wave pulse with TM polarization. As before, the lateral size of the domain is 75 $\mathrm{nm}$, and the incident field is injected through a TF/SF interface. The gold is described by a simple Drude model, and the wavelength range of interest is $[150,600] \mathrm{nm}$. In this problem, the observable of interest is the reflectance, whose analytical solution can be computed with a transfer matrix method. Regarding the DGTD discretization, a $\mathbb{P}_{3}$ polynomial approximation is used along with a fourth-order scheme in time with upwind fluxes $(\alpha=1)$, leading to an overall fourth-order accuracy in both space 
and time. Results are shown in figure 4.3. A perfect match is observed between the numerical and the exact solution, both for normal and oblique incidences.

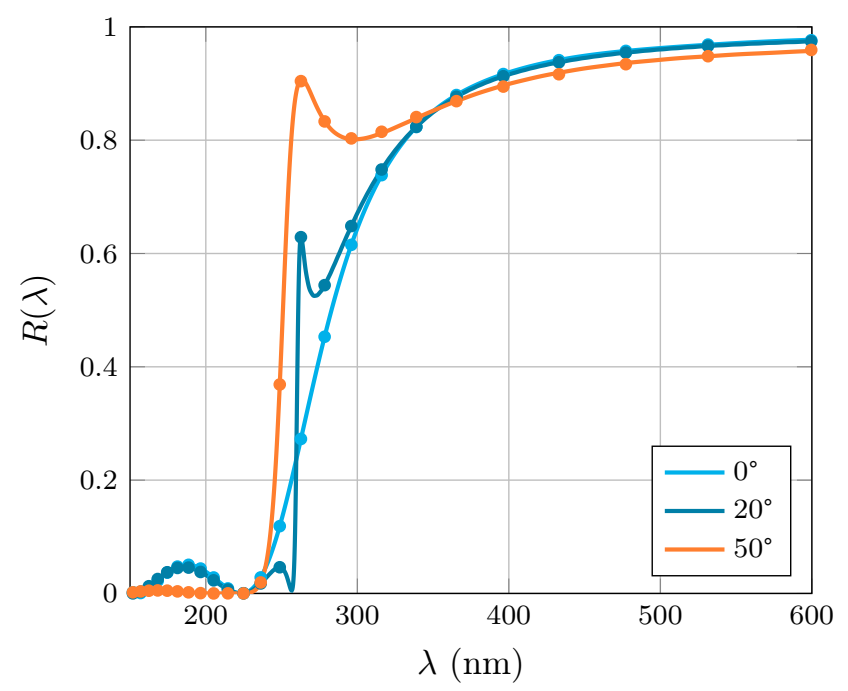

FIGURE 4.3. Reflectance of a bi-periodic $50 \mathrm{~nm}$ thick gold slab under normal and oblique incidence computed with third-order polynomial basis (full line) compared to exact solution (dots).

\subsection{Dielectric color filter}

We now consider optical color filters composed of silicon nanodisks deposited on a glass substrate and embedded in PMMA, as presented in [13] (see figure 4.4). In this contribution, the authors present a set of substractive filters, for which they compute and measure the transmission in normal incidence. In this section, we reproduce the results for a magenta filter under normal incidence, before exploring its response under oblique incidence using the field transform technique, for various angles and polarizations. Results are shown in figure 4.5. As can be seen, at oblique incidences, the filter response is altered: in TM polarization, the filtered wavelength is blue-shifted for $\theta=20^{\circ}$, while the quality factor of the filter is seriously decreased once $40^{\circ}$ of incidence is reached. In TE polarization, the filtered wavelength is red-shifted for $\theta=20^{\circ}$ with new resonances appearing for longer wavelengths. For $\theta=40^{\circ}$, the filter efficiency is seriously compromised. As an illustration, we show in figure 4.6 a field map of the Fourier transform of $E_{x}$ at the operating wavelength of the color filter, illuminated by a monochromatic plane wave with incidence angle $\theta=30^{\circ}$. For clarity, the fields are not represented in the PML regions (at the bottom and the top of the domain).

\subsection{Two dimensional silicon grating}

We now consider a two dimensional silicon grating on a silicon substrate of infinite depth as depicted in figure 4.7. The lateral periodic unit cell length is $d_{\mathrm{G}}=700 \mathrm{~nm}$, the height of the grating $h_{\mathrm{G}}=350$ $\mathrm{nm}$, and the substrate infinitely extended in the negative $z$ direction. For silicon, we used the material parameters from [3]. As for the previous examples, the incident field is injected through a TF/SF interface, on which the reflectance $R$ and diffraction efficiencies $\eta_{n, m}$ are computed. Results of the computation for normal $\left(\theta=0^{\circ}\right)$ and oblique $\left(\theta=20^{\circ}\right)$ incidences are shown in figures 4.9 and 4.8. In normal incidence, it is visible that the normal mode $\eta_{0,0}$ is the only contributor to the reflectance 


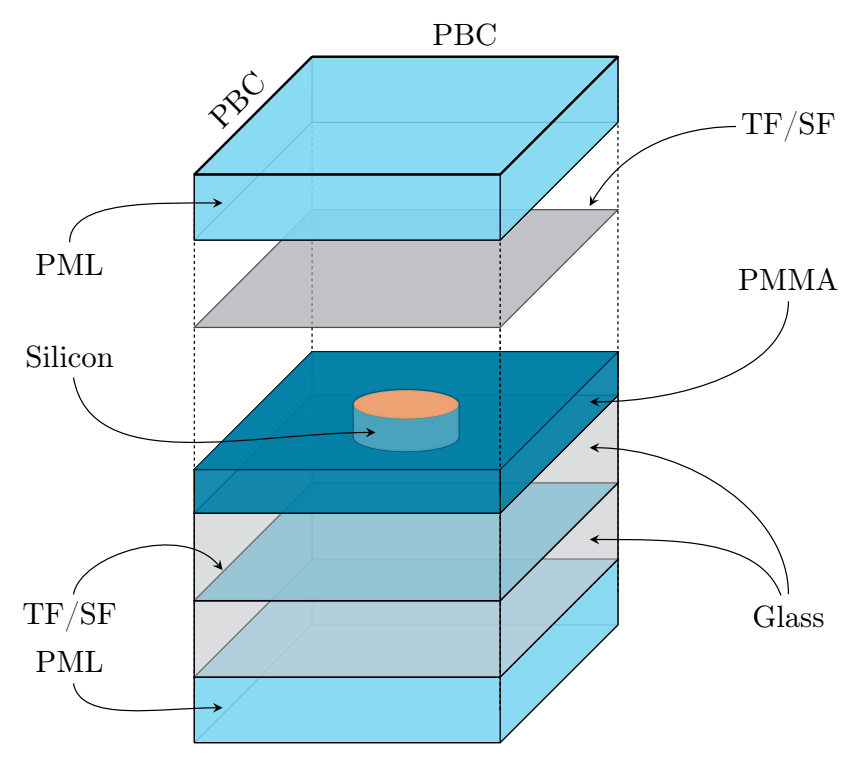

FIgURE 4.4. Silicon-based color filter embedded in PMMA, deposited on a glass substrate. For the magenta filter, the height of the silicon cylinder is $80 \mathrm{~nm}$, and its diameter is $130 \mathrm{~nm}$. The lateral size of the domain is $330 \mathrm{~nm}$. At the bottom, the glass is embedded directly in the PML region, thus mimicking an infinitely thick glass layer.

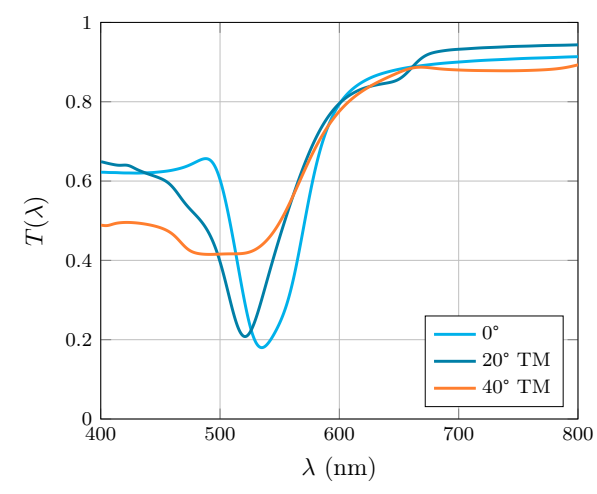

(a) TM polarization

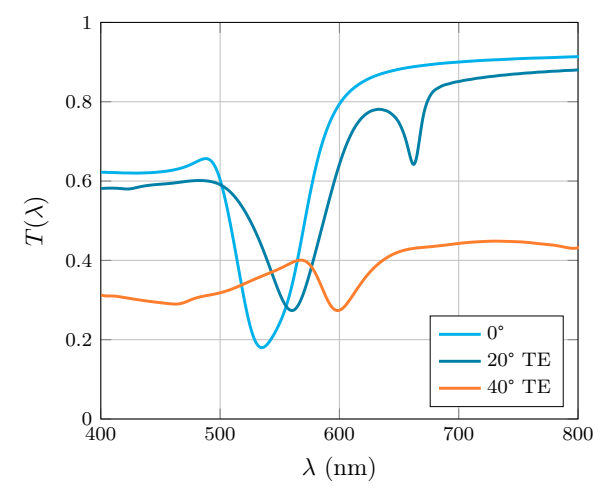

(b) TE polarization

Figure 4.5. Transmission of the magenta color filter at normal and oblique incidences, for both TE and TM polarizations. For both polarizations, the filtering function is altered, either by diminishing its efficiency, or by shifting the filtered wavelength.

above $500 \mathrm{~nm}$. At lower wavelengths, a set of four symmetric high-order diffraction modes appear. As could be expected, the situation is different at oblique incidence, where high-order diffraction modes appear at larger wavelengths. Only two symmetric modes appear below $600 \mathrm{~nm}$, while a higher-order asymmetric mode is present below $480 \mathrm{~nm}$. 


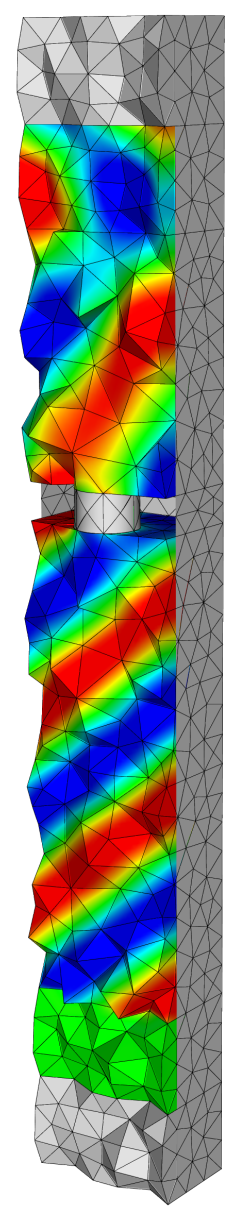

FiguRE 4.6. Field map of the Fourier transform of $E_{x}$ for the color filter setup. The device is illuminated from below by a monochromatic plane wave with incidence angle $\theta=30^{\circ}$.

\section{Conclusions}

In this paper, we complemented the work of [11] on the use of the field transformation technique to handle oblique incidence with regular $\mathrm{PBC}$ on 3D periodic structures in the DGTD framework. The transformed equations were analyzed with the method of characteristics and energy techniques, highlighting several limitations that were then confirmed through numerical experiments. The DGTD discretization of the transformed system was recalled, and the stability of the semi-discrete formulation was proven. Then, insights were given about the effects of the field transformation on the incident field, the observables, and the use of CFS-PMLs in this context. Our implementation was validated on a textbook case, before being applied to the study of two real-life cases: (i) the robustness of a silicon-based color filter against various incidence angles, and (ii) the computation of the efficiency of high-order diffraction modes in a silicon grating. 


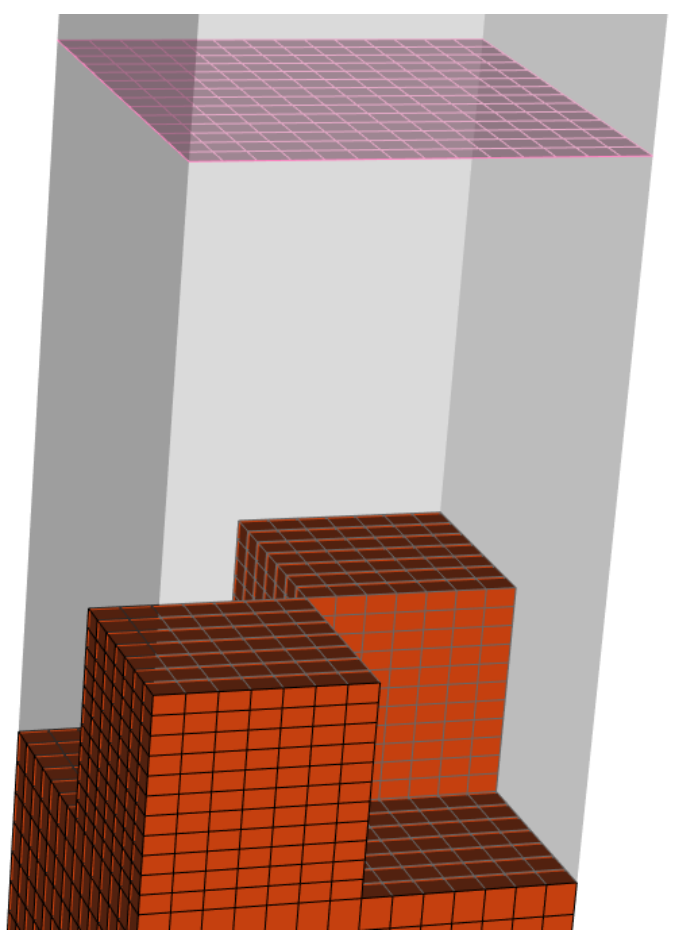

FiguRE 4.7. Computational mesh of the grating unit cell. Gray: periodic boundary faces; pink: TF/SF interface; red: silicon grating on infinite substrate; the remaining domain is air. Both the silicon substrate and the air are truncated by PMLs (not shown here) in the $\hat{\mathbf{z}}$ direction.

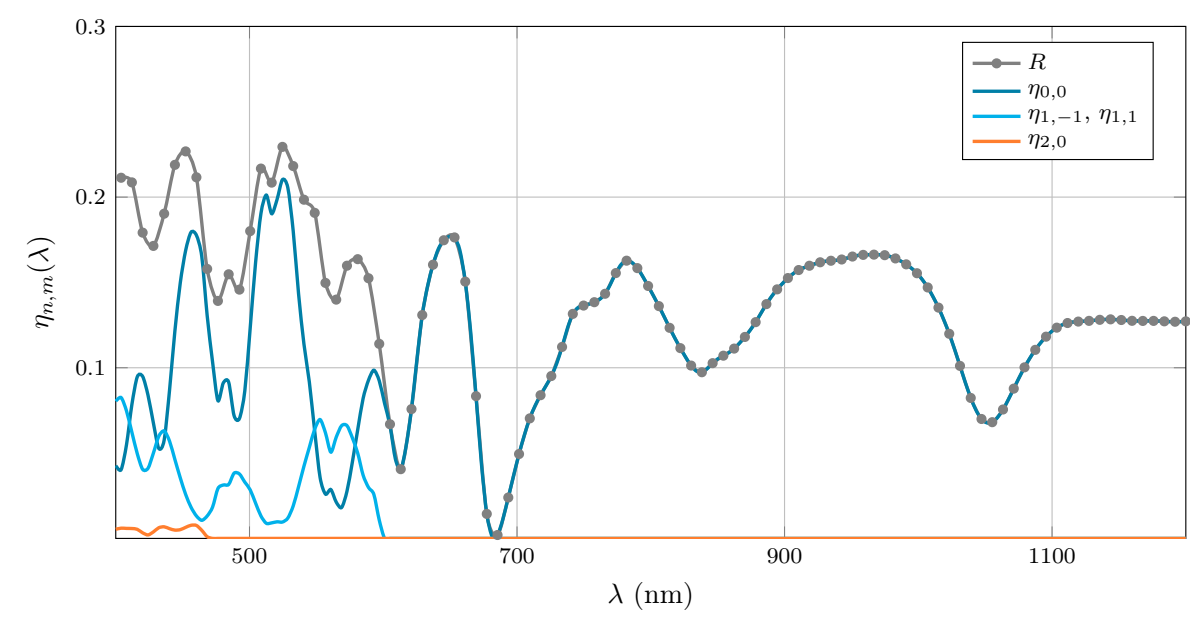

FIgURE 4.8. Diffraction efficiencies of the grating at normal incidence $(\theta=$ $0^{\circ}$ ). The reflectance coefficient is shown in gray dots, while the relevant diffraction efficiencies are shown in blue, cyan and orange. As could be expected, the tilted incidence angle gives rise to different high-order diffraction angles than for the normal incidence case (figure 4.8). Only two symmetric modes appear below $600 \mathrm{~nm}$, and an additional asymmetric mode is also present below $480 \mathrm{~nm}$. 


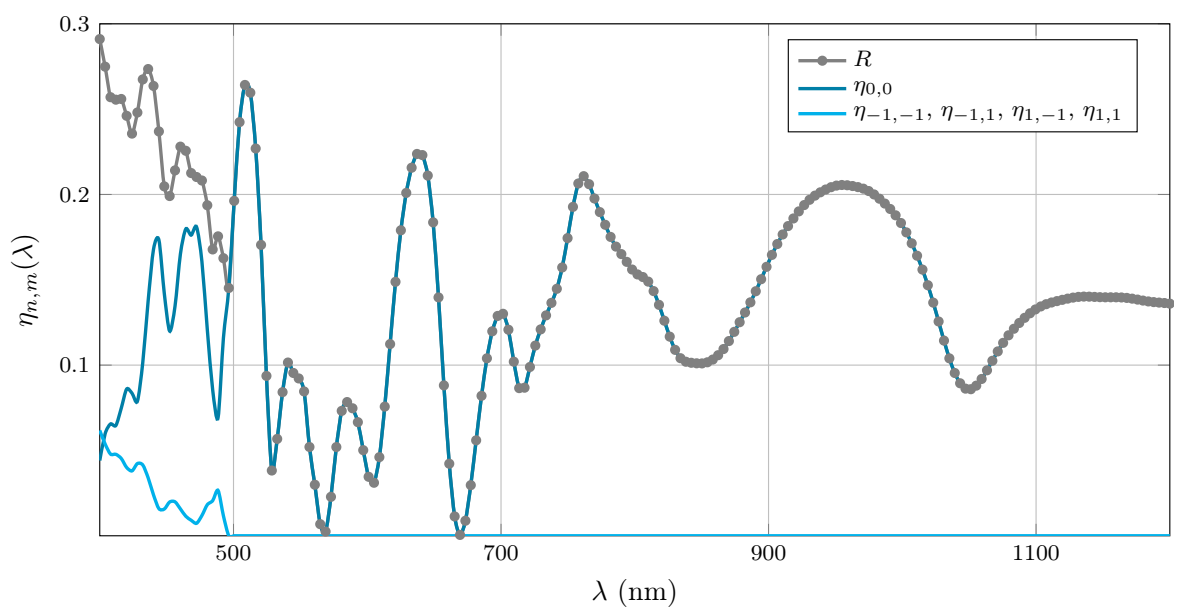

FIGURE 4.9. Diffraction efficiencies of the grating at oblique incidence $(\theta=$ $20^{\circ}$ ). The reflectance coefficient is shown in gray dots, while the relevant diffraction efficiencies are shown in dark and light blue. As can be seen, the normal mode $\eta_{0,0}$ is the only contributor to the reflectance above $500 \mathrm{~nm}$, where a set of four symmetric modes appear.

\section{References}

[1] F. Assous, Jr Ciarlet, P., and S. Labrunie. Mathematical Foundations of Computational Electromagnetism. Springer, 2018.

[2] M. H. Carpenter and C. A. Kennedy. Fourth-order 2n-storage Runge-Kutta schemes. Technical report, National Aeronautics and Space Administration, 1994.

[3] M. A. Green. Self-consistent optical parameters of intrinsic silicon at $300 \mathrm{~K}$ including temperature coefficients. Solar Energy Materials and Solar Cells, 92:1305-1310, 2008.

[4] H. Hotter and H. Steyskal. Broadband FDTD analysis of infinite phased arrays using periodic boundary conditions. Electronics Letters, 35:758-759, 1999.

[5] T. Kokkinos, C. D. Sarris, and G. V. Eleftheriades. Periodic finite-difference time-domain analysis of loaded transmission-line negative-refractive-index metamaterials. IEEE Transactions on Microwave Theory and Techniques, 53:1488-1495, 2005.

[6] M. König. Discontinuous Galerkin Methods in Nanophotonics. PhD thesis, Fakultät für Physik des Karlsruher Instituts für Technologie, 2011. https://publikationen.bibliothek.kit.edu/1000023854.

[7] M. Kuzuoglu and R. Mittra. Frequency dependence of the constitutive parameters of causal perfectly matched layers. IEEE Microwave and Guided Wave Letters, 6:447-449, 1996.

[8] S. Lanteri, C. Scheid, and J. Viquerat. Analysis of a generalized dispersive model coupled to a DGTD method with application to nanophotonics. SIAM J. Sci. Comput., 39:A831-A859, 2017.

[9] K.-L. Lee and P.-K. Wei. Surface plasmon resonance sensing: Periodic metallic nanostructures for highsensitivity biosensing applications. IEEE Nanotechnology Magazine, 10:16-23, 2016.

[10] R. T. Lee and G. S. Smith. A conceptually simple method for incorporating periodic boundary conditions into the FDTD method. Antennas and Propagation Society International Symposium, pages 114-117, 2005.

[11] N. C. Miller, A. D. Baczewski, J. D. Albrecht, and B. Shanker. A discontinuous Galerkin time domain framework for periodic structures subject to oblique excitation. IEEE Transactions on Antennas and Propagation, 62:4386-4391, 2014. 


\section{Simulating 3D PeRiodic StruCtures at oblique incidences}

[12] C. Palmer. Diffraction Grating Handbook. Newport Corporation, sixth edition, 2005.

[13] C.-S. Park, V. R. Shrestha, W. Yue, S. Gao, S.-S. Lee, E.-S. Kim, and D.-Y. Choi. Structural color filters enabled by a dielectric metasurface incorporating hydrogenated amorphous silicon nanodisks. Nature Scientific Reports, 7:2556, 2017.

[14] J. A. Roden, S. D. Gedney, M. P. Kesler, J. G. Maloney, and P. H. Harms. Time-domain analysis of periodic structures at oblique incidence: Orthogonal and nonorthogonal FDTD implementations. IEEE Transactions on Microwave Theory and Techniques, 46:420-427, 1998.

[15] J. S. Shang. Characteristic based methods for the time-domain Maxwell equations, 1993. 29th Aerospace Sciences Meeting, https://arc.aiaa.org/doi/10.2514/6.1991-606.

[16] A. Taflove and S. Hagness. Computational Electrodynamics: The Finite-Difference Time- Domain Method. Artech House, Boston, third edition, 2005.

[17] I. Valuev, A. Deinega, and S. Belousov. Implementation of the iterative finite-difference time-domain technique for simulation of periodic structures at oblique incidence. Computer Physics Communications, 185:1273-1281, 2014.

[18] J. Viquerat. Simulation of electromagnetic waves propagation in nano-optics with a high-order discontinuous Galerkin time-domain method. PhD thesis, INRIA Sophia-Antipolis, 2015. https://hal. archives-ouvertes.fr/tel-01272010/file/main.pdf.

[19] Y. Zeng. Modeling and design of near-field antennas with periodic structures. $\mathrm{PhD}$ thesis, University of Illinois at Urbana-Champaign, 2017.

[20] L. Zou, W. Withayachumnankul, C. Shah, A. Mitchell, M. Bhaskaran, S. Sriram, and C. Fumeaux. Dielectric resonator nanoantennas at visible frequencies. Optics Express, 21:1344-1352, 2013. 